\title{
Universiteit
}

Leiden

The Netherlands

\section{The environment and nature of the Class I protostar Elias 29: Molecular gas observations and the location of ices}

Boogert, A.C.A.; Hogerheijde, M.R.; Ceccarelli, C.; Tielens, A.G.G.M.; Dishoeck, E.F. van;

Blake, G.A.; ... ; Motte, F.

\section{Citation}

Boogert, A. C. A., Hogerheijde, M. R., Ceccarelli, C., Tielens, A. G. G. M., Dishoeck, E. F. van, Blake, G. A., ... Motte, F. (2002). The environment and nature of the Class I protostar Elias 29: Molecular gas observations and the location of ices. Astrophys. J., 570, 708-723. Retrieved from https://hdl.handle.net/1887/2170

Version: $\quad$ Not Applicable (or Unknown)

License:

Downloaded from: https://hdl.handle.net/1887/2170

Note: To cite this publication please use the final published version (if applicable). 


\title{
THE ENVIRONMENT AND NATURE OF THE CLASS I PROTOSTAR ELIAS 29: MOLECULAR GAS OBSERVATIONS AND THE LOCATION OF ICES
}

\author{
A. C. A. Boogert, ${ }^{1}$ M. R. Hogerheijde, ${ }^{2,3}$ C. Ceccarellit, ${ }^{4}$ A. G. G. M. Tielens, ${ }^{5,6}$ \\ E. F. van Dishoeck, ${ }^{7}$ G. A. Blake, ${ }^{8}$ W. B. Latter, ${ }^{9}$ and F. Motte ${ }^{1}$ \\ Received 2001 September 26; accepted 2002 January 16
}

\begin{abstract}
A (sub-)millimeter line and continuum study of the Class I protostar Elias 29 in the $\rho$ Ophiuchi molecular cloud is presented whose goals are to understand the nature of this source and to locate the ices that are abundantly present along this line of sight. Within $15^{\prime \prime}-60^{\prime \prime}$ beams, several different components contribute to the line emission. Two different foreground clouds are detected, an envelope/disk system and a dense ridge of $\mathrm{HCO}^{+}$-rich material. The latter two components are spatially separated in millimeter interferometer maps. We analyze the envelope/disk system by using inside-out collapse and flared disk models. The disk is in a relatively face-on orientation $\left(<60^{\circ}\right)$, which explains many of the remarkable observational features of Elias 29 , such as its flat spectral energy distribution, its brightness in the near-infrared, the extended components found in speckle interferometry observations, and its high-velocity molecular outflow. It cannot account for the ices seen along the line of sight, however. A small fraction of the ices is present in a (remnant) envelope of mass $0.12-0.33 M_{\odot}$, but most of the ices $(\sim 70 \%)$ are present in $\operatorname{cool}(T<40 \mathrm{~K})$ quiescent foreground clouds. This explains the observed absence of thermally processed ices (crystallized $\mathrm{H}_{2} \mathrm{O}$ ) toward Elias 29. Nevertheless, the temperatures could be sufficiently high to account for the low abundance of apolar $\left(\mathrm{CO}, \mathrm{N}_{2}, \mathrm{O}_{2}\right)$ ices. This work shows that it is crucial to obtain spectrally and spatially resolved information from single-dish and interferometric molecular gas observations in order to determine the nature of protostars and to interpret Infrared Space Observatory and future Space Infrared Telescope Facility observations of ices and silicates along a pencil beam.
\end{abstract}

Subject headings: dust, extinction — infrared: ISM — ISM: molecules — stars: formation stars: individual (Elias 29) — submillimeter

\section{INTRODUCTION}

A rich chemical and physical interplay exists between gas and grains in which molecules are formed on grains, creating ice mantles that are preserved in environments ranging from quiescent dense molecular clouds to envelopes and disks around protostars. Various processes, among which are bombardment by cosmic rays, ultraviolet irradiation, heating, and shocks, can physically or chemically alter the icy mantles or return molecules to the gas phase (see Tielens \& Charnley 1997; van Dishoeck \& Blake 1998 and references therein).

A study of the chemical evolution of dense clouds to planet-forming disks would ideally involve observations of molecular gas and ices in a range of environments, from quiescent clouds to disk-dominated protostars. The most pristine, initial conditions are presumably well sampled by

${ }^{1}$ California Institute of Technology, Downs Laboratory of Physics, MS 320-47, Pasadena, CA 91125; boogert@submm.caltech.edu.

${ }^{2}$ Radio Astronomy Laboratory, University of California at Berkeley, Astronomy Department, 601 Campbell Hall 3411, Berkeley, CA 94720.

${ }^{3}$ Current address: Steward Observatory, University of Arizona, 933 North Cherry Avenue, Tucson, AZ 85721.

${ }^{4}$ Observatoire de Bordeaux, 2 Rue de l'Observatoire, BP 89, 33270 Floirac, France.

${ }^{5}$ Kapteyn Astronomical Institute, Postbus Box 800, 9700 AV Groningen, Netherlands.

${ }^{6}$ SRON, Postbus 800, 9700 AV Groningen, Netherlands.

${ }^{7}$ Leiden Observatory, Postbus Box 9513, 2300 RA Leiden, Netherlands.

${ }^{8}$ California Institute of Technology, Division of Geological and Planetary Sciences, MS 150-21, Pasadena, CA 91125.

${ }^{9}$ California Institute of Technology, SIRTF Science Center, IPAC, Pasadena, CA 91125 field stars behind clouds tracing quiescent molecular cloud material (see, e.g., Whittet et al. 1998). Lines of sight to protostars are more difficult to characterize, however, since they may trace quiescent foreground material in addition to the gas and ices in their envelopes and disks (see, e.g., Boogert et al. 2000b). It is thus crucial to characterize the lineof-sight conditions in order to locate the ices and derive physical conditions and eventually the evolution of the molecular gas and solid state in the interstellar medium.

In this paper we study the line of sight of the Class I protostar Elias 29 in the $\rho$ Oph molecular cloud using (sub-)millimeter single-dish and interferometer gas-phase observations. This object is one of the most luminous protostars ( $\sim 36 L_{\odot}$; Chen et al. 1995) in the nearby $\rho$ Oph complex $(d \sim 160 \mathrm{pc}$; Whittet 1974), yet little is known about its nature and line-of-sight conditions. Abundant ice has been detected in its direction (Zinnecker, Webster, \& Geballe 1985; Tanaka et al. 1990). A detailed analysis of ice band profiles indicates that the ices are not strongly thermally processed (i.e., the ices are not crystallized or segregated) despite the presence of abundant warm molecular gas toward the object (Boogert et al. 2000b). This contrasts with high-mass, luminous $\left(>10^{4} L_{\odot}\right)$ protostars, in which significant thermal processing of the ices accompanies the presence of abundant warm molecular gas (Boogert et al. 2000a; van der Tak et al. 2000). The result obtained for Elias 29 can only be understood once the location of the ices and the physical conditions of the various gas components are known. Therefore, in this paper we try to identify any foreground material, the presence of a circumstellar envelope as well as the presence and orientation of a circumstellar disk, and the column density of each component. We will then 
address the question of where the ices are located and what their relation is to the young star. This study will, as a consequence, reveal important information about the nature and evolutionary stage of Elias 29, which has many interesting and unique properties (Elias 1978).

Details of the single-dish and interferometer observations are presented in $\S 2$, and the maps and spectra are decomposed and interpreted in $\S 3$. The physical conditions are determined for the different components along the line of sight, among which are two foreground clouds (§ 4.1), a remnant envelope and face-on disk ( $(4.2)$, as well as a dense ridge from which Elias 29 probably formed ( $\$ 4.3$ ). The gasphase conditions and abundances are linked to the ice observations in $\S 4.4$. The depletion of gas-phase species is compared to young Class 0 objects and quiescent clouds in $\S 4.5$. The results are summarized in $\S 5$.

\section{OBSERVATIONS}

Single-dish and interferometer millimeter wave spectral line and continuum observations were made toward the infrared position of Elias $29 \quad\left(\alpha=16^{\mathrm{h}} 27^{\mathrm{m}} 09 \mathrm{~s} .5\right.$, $\left.\delta=-24^{\circ} 37^{\prime} 18^{\prime \prime}[\mathrm{J} 2000.0]\right)$. Rotational lines of $\mathrm{CO}, \mathrm{HCO}^{+}$, $\mathrm{CS}, \mathrm{H}_{2} \mathrm{CO}$, and $\mathrm{CH}_{3} \mathrm{OH}$ and isotopes were selected in the 70-400 GHz spectral range based on their sensitivity to column density, temperature, and density (Blake et al. 1995). Below we discuss the technical details of these observations. Not discussed is a map of the CO 6-5 line $(692 \mathrm{GHz})$ for which we refer to C. Ceccarelli et al. (2002, in preparation). Also, the technical details of the $1.3 \mathrm{~mm}$ IRAM $30 \mathrm{~m}$ continuum map at $15^{\prime \prime}$ spatial resolution that is used in this paper are discussed elsewhere (Motte, André, \& Neri 1998). Finally, the infrared spectral energy distribution (SED) that we use consists of 2-45 and 45-200 $\mu \mathrm{m}$ spectra that were obtained with the Infrared Space Observatory (ISO) shortwavelength spectrometer (SWS) and long-wavelength spectrometer (LWS) instruments in apertures of $\sim 25^{\prime \prime}$ and $\sim 80^{\prime \prime}$, respectively, and were extensively discussed in Boogert et al. (2000b). A summary of all the observations used in this paper is given in Table 1.

\subsection{Single-Dish 70-400 GHz Spectral Line Observations}

Single-dish NRAO 12 m, James Clerk Maxwell Telescope (JCMT), and Caltech Submillimeter Observatory (CSO) millimeter wave observations were made with a single pointing toward Elias 29 during a number of observing runs in the 1995-2001 period (Table 1). In some lines, small maps were made of the Elias 29 environment with the CSO. At the JCMT and CSO, we used the Digital Autocorrelation Spectrometer (DAS) and acousto-optical spectrometer (AOS) back ends in the highest available spectral resolution mode $\left(\sim 0.1 \mathrm{~km} \mathrm{~s}^{-1}\right)$, in the $200-400 \mathrm{GHz}$ spectral range. At the NRAO $12 \mathrm{~m}$ telescope, we used the autocorrelator back end, with a channel width of $0.049 \mathrm{MHz}$, resulting in Nyquist-sampled spectra with resolutions of $0.40-0.20 \mathrm{~km}$ $\mathrm{s}^{-1}$ at the frequencies $70-145 \mathrm{GHz}$.

For low-frequency transitions that trace extended cloud structure, we took $\alpha=16^{\mathrm{h}} 23^{\mathrm{m}} 01^{\mathrm{s}} .5, \quad \delta=-24^{\circ} 36^{\prime} 58^{\prime \prime}$ (J2000.0) as an off position, which is found to have very little or no ${ }^{13} \mathrm{CO}$ emission (Loren 1989). For higher frequency $\mathrm{CO}$ transitions, we took an off position of $2700^{\prime \prime}$ in azimuth, and for other molecules, the azimuth offset was $900^{\prime \prime}$. No contamination by emission in the off position is evident in our spectra.

Our line observations were corrected for atmospheric attenuation and telescope losses using the standard chopping wheel technique (Kutner \& Ulich 1981). The NRAO 12 $\mathrm{m}$ data retrieved from the telescope are on a $T_{R}^{*}$ scale, and the CSO and JCMT data are in $T_{A}^{*}$. To convert to the mainbeam brightness temperature $\left(T_{\mathrm{mb}}\right)$, we divide by the beam efficiencies:

$$
\begin{aligned}
& T_{\mathrm{mb}}=T_{R}^{*} / \eta_{m}^{*}(\mathrm{NRAO} 12 \mathrm{~m}) \\
& T_{\mathrm{mb}}=T_{A}^{*} / \eta_{\mathrm{mb}}(\mathrm{JCMT}, \mathrm{CSO}) .
\end{aligned}
$$

This corrects for losses in the beam sidelobes. For the JCMT and $\mathrm{CSO}$, losses due to forward spillover and scattering are included in $\eta_{\mathrm{mb}}$. The beam efficiencies applied to our data are summarized in Table 2. For the CSO telescope,${ }^{10} \eta_{\mathrm{mb}}$ and the main-beam size (Table 3) were determined by observing Mars during observing runs in 1999 March and 2001 January. For the NRAO $12 \mathrm{~m}$ telescope, ${ }^{11}$ we used the

\footnotetext{
${ }^{10} \mathrm{CSO}$ beam parameters are available at http://www.submm.caltech.edu/cso/receivers/beams.html.

${ }_{11}$ User's Manual for the NRAO-12 m Millimeter-Wave Telescope (Mangum 1999) is available at http://www.tuc.nrao.edu/12meter/ obsinfo.html.
}

TABLE 1

\begin{tabular}{|c|c|c|c|c|}
\hline Telescope & $\begin{array}{l}\text { Beam Size } \\
(\operatorname{arcsec})\end{array}$ & $\lambda$ or $\nu$ & Species $^{\mathrm{a}}$ & Date or Reference \\
\hline NRAO $12 \mathrm{~m} \ldots . . . .$. & $43-86$ & $70-145 \mathrm{GHz}$ & $\mathrm{CO}, \mathrm{CS}, \mathrm{HCO}^{+}, \mathrm{H}_{2} \mathrm{CO}, \mathrm{CH}_{3} \mathrm{OH}$ & 1995 May \\
\hline JCMT ................ & $14-22$ & $200-400 \mathrm{GHz}$ & $\mathrm{CO}, \mathrm{CS}, \mathrm{HCO}^{+}, \mathrm{H}_{2} \mathrm{CO}, \mathrm{CH}_{3} \mathrm{OH}$ & $1995-1997$ \\
\hline JCMT …............. & 7 & $692 \mathrm{GHz}$ & $\mathrm{CO} 6-5$ & C. Ceccarelli et al. 2002 , in preparation \\
\hline $\mathrm{CSO} \ldots \ldots \ldots \ldots \ldots \ldots$ & $21-35$ & $200-400 \mathrm{GHz}$ & $\mathrm{H}_{2} \mathrm{CO}, \mathrm{CH}_{3} \mathrm{OH}, \mathrm{CO}, \mathrm{HCO}^{+}$maps & 1999-2001 \\
\hline OVRO .................. & $4 \times 8,3 \times 6$ & $87,110 \mathrm{GHz}$ & $\mathrm{CO}, \mathrm{HCO}^{+}, \mathrm{SiO}$ & 1999 Sep-2000 July \\
\hline OVRO .................. & $4 \times 8,3 \times 6$ & $2.7,3.3 \mathrm{~mm}$ & Continuum & 1999 Sep-2000 Jul \\
\hline IRAM $30 \mathrm{~m} . . . \ldots . .$. & 15 & $1.3 \mathrm{~mm}$ & Continuum & Motte et al. 1998 \\
\hline ISO SWS.............. & $14-33$ & $2.3-45 \mu \mathrm{m}$ & $\begin{array}{l}\text { Continuum } \\
\text { Ices, silicates }\end{array}$ & Boogert et al. 2000b \\
\hline ISO LWS ............. & 80 & $45-200 \mu \mathrm{m}$ & Continuum & Boogert et al. 2000b \\
\hline
\end{tabular}

ObServational Summary

a Main species and/or isotopes measured. For more details, see Table 3. 
TABLE 2

Applied Beam EfFiciencies

\begin{tabular}{ccc}
\hline \hline Telescope & $\begin{array}{c}\text { Frequency } \\
(\mathrm{GHz})\end{array}$ & $\eta_{\mathrm{mb}}{ }^{\mathrm{a}}$ \\
\hline NRAO $12 \mathrm{~m} \ldots \ldots . .$. & $70-85$ & 1.00 \\
& $85-90$ & 0.95 \\
& $95-115$ & 0.93 \\
& $140-150$ & 0.81 \\
JCMT ............... & $200-270$ & 0.68 \\
& $320-380$ & 0.60 \\
CSO .................. & $200-270$ & $0.76^{\mathrm{b}}$ \\
& $320-380$ & $0.78^{\mathrm{b}}$ \\
\hline
\end{tabular}

a Here $\eta_{m}^{*}$ is given for NRAO $12 \mathrm{~m}$.

${ }^{\mathrm{b}}$ For 1999 March; 10\% lower in 2001 January.

theoretical values of $\eta_{m}^{*}$ from the Ruze equation, increased by a small factor $(\sim 1.08)$ needed to reproduce the experimental values (Mangum 1999). As a check, we determined $\eta_{m}^{*}$ from our observation of Jupiter, yielding $\eta_{m}^{*}=0.80$ at
$145 \mathrm{GHz}$, in excellent agreement with the assumed value. For the JCMT, the efficiency factors are taken from regularly measured values reported on the JCMT Web site. ${ }^{12}$

The calibration accuracy between different observing runs is not expected to be better than $25 \%$ (see, e.g., Mangum 1993). As a check on the calibration accuracy, we observed the very bright nearby source IRAS 16293-2422 during our JCMT and CSO runs. We find that line intensities, observed with the same telescope during the night and in separate observing runs, indeed scatter with $20 \%-30 \%$ variations around the mean. Similar differences are found when comparing JCMT observations to the standard spectra available at the JCMT Web site. In this paper, we will assume an uncertainty of $25 \%$ in the line intensity unless noted otherwise.

The data were reduced with the GILDAS/CLASS reduction package, applying low-order baselines and using

12 JCMT beam parameters are available at http://www.jach.hawaii.edu/JACpublic/JCMT

TABLE 3

Rotational Transitions Observed toward Elias 29 (Center Position) with Single-Dish Telescopes

\begin{tabular}{|c|c|c|c|c|c|c|c|c|c|}
\hline Molecule & Transition & $\begin{array}{c}\text { Frequency } \\
(\mathrm{MHz})\end{array}$ & $\begin{array}{l}T_{\mathrm{MB}}{ }^{\mathrm{a}} \\
(\mathrm{K})\end{array}$ & $\begin{array}{r}\int T_{\mathrm{MB}} d v^{\mathrm{a}} \\
\left(\mathrm{K} \mathrm{km} \mathrm{s}^{-1}\right)\end{array}$ & $\begin{array}{l}\text { FWHM } \\
\left(\mathrm{km} \mathrm{s}^{-1}\right)\end{array}$ & $\begin{array}{c}V_{\mathrm{LSR}} \\
\left(\mathrm{km} \mathrm{s}^{-1}\right)\end{array}$ & $\begin{array}{c}\text { Beam Size } \\
(\operatorname{arcsec})\end{array}$ & Telescope & Date \\
\hline \multirow[t]{4}{*}{$\mathrm{CO} \ldots \ldots \ldots \ldots$} & $2-1$ & $230,538.0$ & 15.9 & 19.4 & 6 & $2 / 6.5$ & 21 & JCMT & 1997 Mar \\
\hline & $3-2$ & $345,796.0$ & 26 & 98 & 7 & $2 / 6.5$ & 14 & JCMT & $1996 \mathrm{Feb}$ \\
\hline & & & 9 & 46 & 6 & $2 / 6.5$ & 21 & $\mathrm{CSO}$ & $2000 \mathrm{Jul}$ \\
\hline & $6-5^{\mathrm{b}}$ & $691,473.0$ & 17 & 124 & 12 & $1.8 / 6.5$ & 7 & JCMT & $1995 \mathrm{Apr}$ \\
\hline${ }^{13} \mathrm{CO} \ldots \ldots \ldots$. & $6-5^{\mathrm{b}}$ & $661,067.4$ & 10 & 39 & 3.6 & 4.78 & 7 & JCMT & $1995 \mathrm{Apr}$ \\
\hline \multirow[t]{4}{*}{$\mathrm{C}^{17} \mathrm{O} \ldots \ldots \ldots$} & $1-0$ triplet & $112,358.7$ & 0.40 & 0.99 & 2.3 & & 56 & NRAO & 1995 May \\
\hline & & $112,359.0$ & 0.94 & 1.00 & 1.0 & 3.58 & & NRAO & 1995 May \\
\hline & & $112,360.0$ & 0.68 & 1.05 & 1.5 & & & NRAO & 1995 May \\
\hline & 2-1 multiplet & $224,714.3$ & 1.76 & 4.95 & 2.63 & 4.08 & 22 & JCMT & $1995 \mathrm{Mar}$ \\
\hline \multirow{4}{*}{$\mathrm{C}^{18} \mathrm{O} \ldots \ldots \ldots$} & $1-0$ & $109,782.2$ & 6.34 & 11.0 & 2.0 & 3.6 & 57 & NRAO & 1995 May \\
\hline & $2-1$ & $219,560.4$ & 5.81 & 16.2 & 2.62 & 4.19 & 22 & JCMT & $1995 \mathrm{Mar}$ \\
\hline & & & 9.6 & 21.3 & 2.41 & 4.05 & 35 & $\mathrm{CSO}$ & 2001 Jan \\
\hline & $3-2$ & $329,330.6$ & 4.3 & 10.4 & 2.28 & 4.24 & 15 & JCMT & $1996 \mathrm{Feb}$ \\
\hline \multirow[t]{3}{*}{ CS ................... } & $2-1$ & $97,981.0$ & 1.28 & 3.55 & 2.61 & 3.96 & 64 & NRAO & 1995 May \\
\hline & $5-4$ & $244,935.6$ & 0.56 & 0.94 & 1.56 & 4.92 & 20 & JCMT & $1995 \mathrm{Mar}$ \\
\hline & $7-6$ & $342,883.0$ & $<0.09$ & $<0.13$ & $\ldots$ & $\ldots$ & 14 & JCMT & 1996 Feb \\
\hline $\mathrm{C}^{34} \mathrm{~S} \ldots \ldots \ldots$ & $2-1$ & $96,412.9$ & 0.14 & 0.23 & 1.62 & 3.29 & 65 & NRAO & 1995 May \\
\hline \multirow[t]{4}{*}{$\mathrm{p}-\mathrm{H}_{2} \mathrm{CO} \ldots$} & $1_{01}-0_{00}$ & $72,838.0$ & 0.62 & 1.44 & 2.19 & $3.80 / 5.39$ & 86 & NRAO & 1995 May \\
\hline & $2_{02}-1_{01}$ & $145,603.0$ & 0.56 & 0.89 & 2.0 & $3.67 / 4.10$ & 43 & NRAO & 1995 May \\
\hline & $3_{03}-2_{02}$ & $218,222.2$ & 0.39 & 0.66 & 1.89 & 4.60 & 22 & JCMT & 1995 Mar \\
\hline & $3_{22}-2_{21}$ & $218,475.6$ & $<0.06$ & $\ldots$ & $\ldots$ & $\ldots$ & 22 & JCMT & $1995 \mathrm{Mar}$ \\
\hline \multirow[t]{4}{*}{$\mathrm{o}-\mathrm{H}_{2} \mathrm{CO} \ldots$} & $2_{12}-1_{11}$ & $140,839.5$ & 0.80 & 1.85 & 2.17 & 4.20 & 45 & NRAO & 1995 May \\
\hline & $2_{11}-1_{10}$ & $150,498.4$ & 0.80 & 1.85 & 2.17 & 4.20 & 42 & NRAO & 1995 May \\
\hline & $3_{12}-2_{11}$ & $225,697.8$ & 0.41 & 0.76 & 1.76 & 4.93 & 21 & JCMT & $1995 \mathrm{Mar}$ \\
\hline & & & 0.26 & 0.48 & 1.70 & 4.51 & 32 & $\mathrm{CSO}$ & 2000 Jul \\
\hline \multirow[t]{5}{*}{$\mathrm{CH}_{3} \mathrm{OH} \ldots .}$. & 2-1 triplet & $96,739.4$ & 0.15 & 0.19 & 1.24 & $\ldots$ & 65 & NRAO & 1995 May \\
\hline & & $96,741.4$ & 0.18 & 0.33 & 1.68 & 3.59 & & NRAO & 1995 May \\
\hline & & $96,744.6$ & $<0.03$ & $\ldots$ & $\ldots$ & $\ldots$ & & NRAO & 1995 May \\
\hline & 5-4 multiplet & $241,802.0$ & $<0.05$ & $\ldots$ & $\ldots$ & $\ldots$ & 20 & JCMT & $1997 \mathrm{Mar}$ \\
\hline & & & $<0.02$ & & $\ldots$ & $\ldots$ & 31 & $\mathrm{CSO}$ & 2000 Jun \\
\hline \multirow[t]{4}{*}{$\mathrm{HCO}^{+} \ldots \ldots$} & $1-0$ & $89,188.5$ & 3.57 & 5.69 & $3.3 / 1.0$ & $4.3 / 4.7$ & 70 & NRAO & 1995 May \\
\hline & $3-2$ & $267,557.6$ & 1.06 & 2.7 & 2.28 & 4.77 & 18 & JCMT & 1996 May \\
\hline & & & 4.34 & 4.9 & 1.05 & 4.63 & 28 & $\mathrm{CSO}$ & 2000 Jun \\
\hline & $4-3$ & $356,734.3$ & 0.30 & 0.80 & 2.5 & 4.5 & 21 & $\mathrm{CSO}$ & $1999 \mathrm{Mar}$ \\
\hline \multirow[t]{2}{*}{$\mathrm{H}^{13} \mathrm{CO}^{+} \ldots$} & $1-0$ & $86,754.3$ & 0.38 & 0.36 & 0.90 & 4.66 & 72 & NRAO & 1995 May \\
\hline & $3-2$ & $260,255.5$ & $0.04(0.02)$ & $0.09(0.02)$ & 2.15 & 4.89 & 29 & $\mathrm{CSO}$ & 1999 Mar \\
\hline
\end{tabular}

${ }^{a}$ Calibration errors are $25 \%$ unless noted otherwise in parentheses.

b CO 6-5 spectra presented and analyzed in detail in C. Ceccarelli et al. 2002, in preparation. 
weighted means in averaging individual spectra. The reduced single-dish spectra and maps are presented in Figures $1-3$ and Table 3 and analyzed in $\S 3.1$.

\subsection{Interferometer Spectral Line and Continuum Observations}

Elias 29 was observed with the Owens Valley Radio Observatory (OVRO) millimeter array in the 1999/2000 season (Table 1). The low elevation of the source allowed for only short tracks $(\sim 7 \mathrm{hr})$. The digital correlator system was used in the " 182 " mode, providing simultaneous observations of the $\mathrm{C}^{18} \mathrm{O} 1-0$ and ${ }^{13} \mathrm{CO} 1-0$ lines in one local oscillator setting as well as the $\mathrm{HCO}^{+} 1-0$ and $\mathrm{SiO} 2-1$ lines in another. The spectral resolution is $0.35 \mathrm{~km} \mathrm{~s}^{-1}$, with a velocity coverage of $20 \mathrm{~km} \mathrm{~s}^{-1}$. The telescope configurations $\mathrm{L}, \mathrm{H}$, and $\mathrm{E}$ were used in all lines as well as the continuum. The weather conditions were best during the $\mathrm{L}$ and $\mathrm{H}$ observations, resulting in $T_{\mathrm{sys}} \sim 500 \mathrm{~K}$ at $110 \mathrm{GHz}$ and $800 \mathrm{~K}$ at $87 \mathrm{GHz}$. System temperatures were worse in the E track: 700 and $1300 \mathrm{~K}$, respectively. The data were reduced in a standard way in the OVRO/MMA reduction package. The nearby source NRAO 530 was used as a gain calibrator, and flux calibration was performed on the planets Uranus and Neptune. The calibrated tracks were combined, and deconvolved images were constructed with the MIRIAD software package using uniform weighting. The resulting synthesized elliptical beam sizes are $4^{\prime \prime} \times 8^{\prime \prime}$ at $87 \mathrm{GHz}$ and $3^{\prime \prime} \times 6^{\prime \prime}$ at $110 \mathrm{GHz}$; the noise levels achieved per channel in the final data are 0.26 and $0.13 \mathrm{Jy}$ beam $^{-1}$, respectively. Strong and extended ${ }^{13} \mathrm{CO}$ and $\mathrm{HCO}^{+} 1-0$ emission is detected with perhaps a weak detection of $\mathrm{C}^{18} \mathrm{O} 1-0$ (Figs. 4-6). SiO 2-1 is undetected toward Elias 29.

No obvious strong continuum source was seen in the OVRO 2.7 and $3.4 \mathrm{~mm}$ maps. A weak $3 \sigma$ peak $(7 \mathrm{mJy})$ is detected at $3.4 \mathrm{~mm}$. This is the strongest peak in the map, and it is centered on the infrared position of Elias 29. At 2.7 $\mathrm{mm}$ a peak of only $2 \sigma(5 \mathrm{mJy})$ significance appears at the same position. For our analysis, we take a weighted mean of these values, which we will refer to as the continuum flux at $3.0 \mathrm{~mm}: F(3 \mathrm{~mm})=6.1 \pm 1.7 \mathrm{mJy}$. This is only a $3.5 \sigma$ result and needs confirmation by additional observations.

\section{RESULTS}

\subsection{Large-Scale Emission: Single-Dish Spectra}

The single-dish maps show that Elias 29 is not a particularly prominent center of molecular line emission. Within a radius of $\sim 1 ! 5$, the $\mathrm{HCO}^{+} 3-2$ and $\mathrm{C}^{18} \mathrm{O} 2-1$ emission is highly structured and quite differently distributed (Figs. 1 and 2).

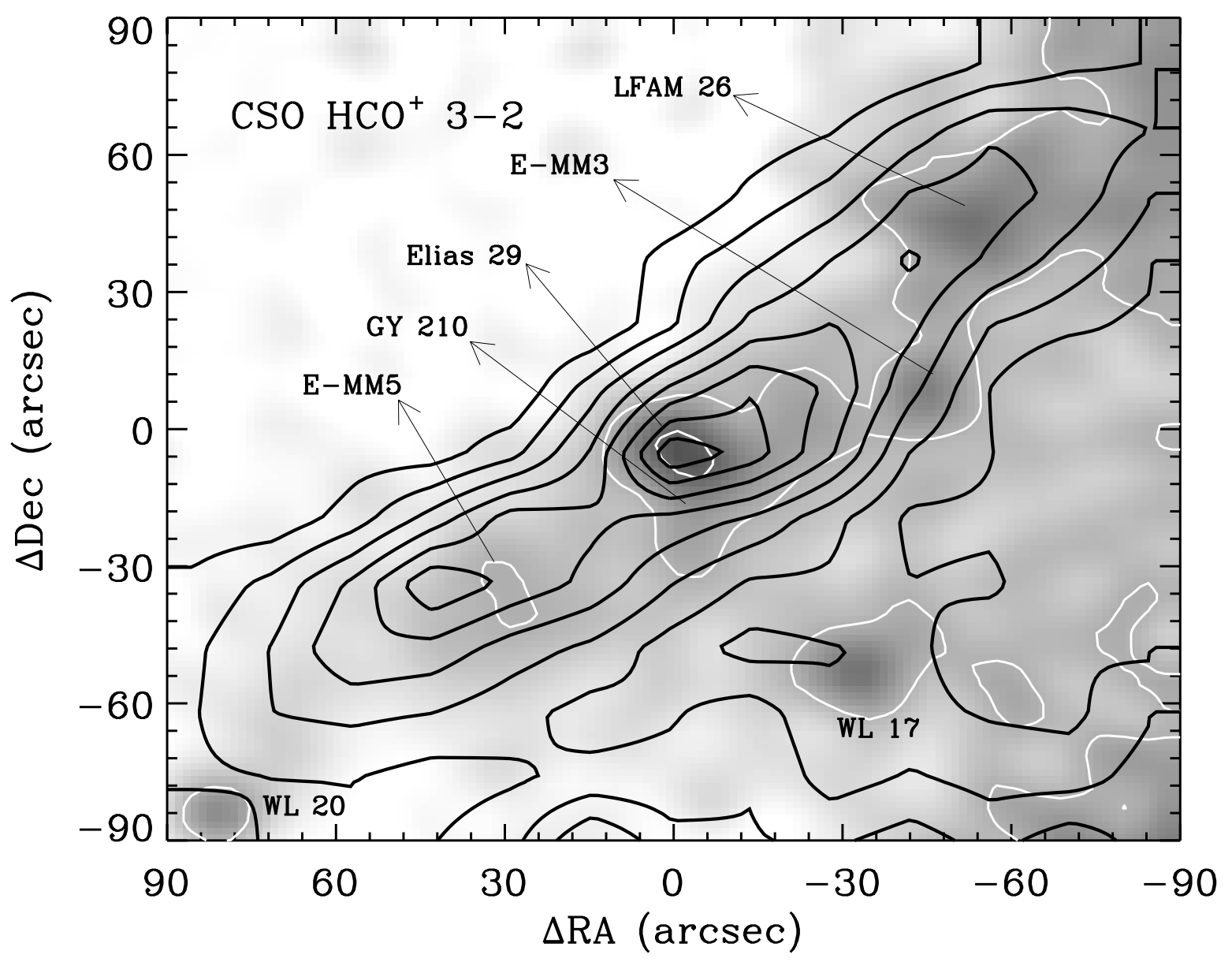

FIG. 1.-Overlay of IRAM $30 \mathrm{~m} 1.3 \mathrm{~mm}$ continuum map (gray scale and two white contours; Motte et al. 1998) and integrated $\mathrm{CSO}^{-} \mathrm{HCO}^{+} 3-2$ emission (black contours) of the Elias 29 region with source identifications. Contour levels for the continuum map are at the 7 and $14 \sigma$ confidence level, with $\sigma=10 \mathrm{mJy}$ per $15^{\prime \prime}$ beam, and $\int T_{\mathrm{MB}} d v=1.1,1.6, \ldots, 4.6 \mathrm{~K} \mathrm{~km} \mathrm{~s}^{-1}$ for $\mathrm{HCO}^{+} 3-2\left(\sigma=0.2 \mathrm{~K} \mathrm{~km} \mathrm{~s}^{-1}\right)$. The $1.3 \mathrm{~mm}$ map was aligned to the radio continuum positions of Elias 29 and WL 20 . The $\mathrm{HCO}^{+}$map in turn was aligned to the $1.3 \mathrm{~mm}$ map, for which a small shift of $5^{\prime \prime}$ to the southwest was needed. 

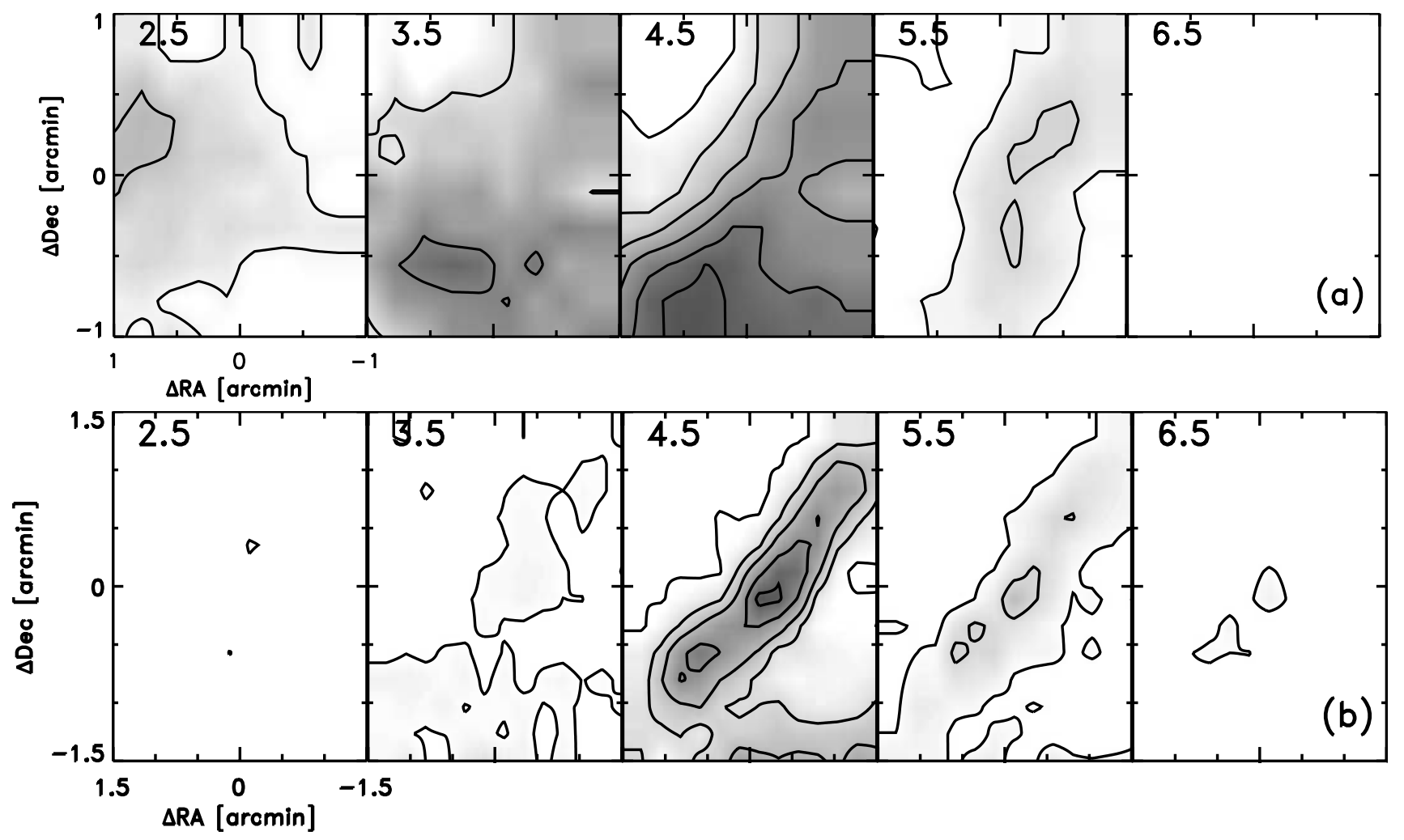

FIG. 2.- Channel maps of $(a) \mathrm{C}^{18} \mathrm{O} 2-1$ and $(b) \mathrm{HCO}^{+} 3-2$ emission in the Elias 29 region, observed with the CSO telescope. The emission is integrated over a $1 \mathrm{~km} \mathrm{~s}^{-1}$ interval, with the central velocity (in units of $\mathrm{km} \mathrm{s}^{-1}$ ) indicated in the upper left-hand corners. The contour levels are equally spaced $\int T_{\mathrm{MB}} d v=0.8$, $2.1, \ldots, 9.9 \mathrm{~K} \mathrm{~km} \mathrm{~s}^{-1}$ for $\mathrm{C}^{18} \mathrm{O}\left(\sigma=0.20 \mathrm{~K} \mathrm{~km} \mathrm{~s}^{-1}\right)$, and $0.3,0.9, \ldots, 2.7 \mathrm{~K} \mathrm{~km} \mathrm{~s}^{-1}$ for $\mathrm{HCO}^{+}\left(\sigma=0.09 \mathrm{~K} \mathrm{~km} \mathrm{~s}^{-1}\right)$. Note that the map scale is $2^{\prime} \times 2^{\prime}$ for $\mathrm{C}^{18} \mathrm{O}$ and $3^{\prime} \times 3^{\prime}$ for $\mathrm{HCO}^{+}$. Foreground clouds are seen in $\mathrm{C}^{18} \mathrm{O} 2-1$ at 2.5 and $3.7 \mathrm{~km} \mathrm{~s}^{-1}$. The Elias 29 core and surrounding ridge are seen at higher velocities, especially in $\mathrm{HCO}^{+}$.

The $\mathrm{HCO}^{+} 3-2$ emission, tracing high densities $\left(\geq 10^{5}\right.$ $\left.\mathrm{cm}^{-3}\right)$, is concentrated in a remarkable ridgelike structure, oriented in the southeast-northwest direction (Fig. 1) at a velocity of $V_{\mathrm{LSR}} \sim 5.0 \mathrm{~km} \mathrm{~s}^{-1}$ (Fig. 2). It is likely no coincidence that the protostars Elias 29, WL 20, LFAM 26, and GY 210 as well as the $1.3 \mathrm{~mm}$ continuum protostellar condensations E-MM3 and E-MM5 are all located along this dense ridge (Fig. 1). The ridge is also particularly prominent in the $800 \mu \mathrm{m}$ continuum (Johnstone et al. 2000b). Star formation along dense filamentary structures is common in the $\rho$ Oph cloud and has been explained by the presence of magnetic field tubes or, more likely, by externally induced shocks (see Motte et al. 1998 for a short discussion).

The $\mathrm{C}^{18} \mathrm{O} 2-1$ emission, a column rather than volume density tracer, shows that at least three clouds are present along the line of sight of Elias 29 (Fig. 2). The channel maps show a cloud at $V_{\mathrm{LSR}} \sim 2.7 \mathrm{~km} \mathrm{~s}^{-1}$ that peaks to the northeast of Elias 29 and a cloud at $V_{\mathrm{LSR}} \sim 3.8 \mathrm{~km} \mathrm{~s}^{-1}$ spread rather evenly over the map. The brightest cloud at $\sim 5 \mathrm{~km}$ $\mathrm{s}^{-1}$ peaks prominently near the south-southwest of the map and is probably that in which the dense $\mathrm{HCO}^{+}$ridge resides given the similar velocities. All these clouds are likely present in the foreground since absorption in the ${ }^{12} \mathrm{CO}$ emission lines is seen at these velocities (Fig. 3).

In various other single-dish lines these clouds show up as discrete emission components (Fig. 3). The 2.7 and $3.8 \mathrm{~km}$ $\mathrm{s}^{-1}$ components are primarily seen in the low-lying transitions of the $\mathrm{CS}, \mathrm{HCO}^{+}, \mathrm{H}_{2} \mathrm{CO}$, and $\mathrm{CH}_{3} \mathrm{OH}$ molecules, indicating low densities and temperatures of these extended clouds. The emission at $5.0 \mathrm{~km} \mathrm{~s}^{-1}$, identified with the Elias 29 core and ridge, shows up in the high-excitation lines.

Given the fact that these emission components have different spatial distributions, their relative intensity also depends on beam size. The $\mathrm{HCO}^{+} 3-2$ line is a factor of 4 stronger and a factor of 2 narrower in the CSO data compared to JCMT: the larger CSO beam (28" vs. $18^{\prime \prime}$; Table 3) picks up more emission from the ridge, which is bright in the west ( $\$ 3.2$ ). The wide-beam NRAO $12 \mathrm{~m} \mathrm{HCO}^{+} 1-0$ and $\mathrm{H}^{13} \mathrm{CO}^{+} 1-0$ spectra have remarkably narrow $5.0 \mathrm{~km} \mathrm{~s}^{-1}$ components, originating from the extended ridge. In contrast, the $\mathrm{H}_{2} \mathrm{CO} 3_{12}-2_{11}$ line may peak on the Elias 29 core rather than the ridge. It has the same width in the CSO and JCMT beams, but the CSO spectrum is weaker (Table 3 ).

We have performed a Gaussian decomposition of the emission lines to separate the foreground clouds from the dense material around Elias 29. The derived relative strength of these blended lines depends sensitively on the assumed line width. The most reasonable solution, based on the $\mathrm{H}_{2} \mathrm{CO}$ and $\mathrm{CS}$ lines and the $\mathrm{C}^{18} \mathrm{O}$ map, is to simultaneously fit the peak intensity with three Gaussians, centered on $V_{\mathrm{LSR}}=2.7,3.8$, and $5.0 \mathrm{~km} \mathrm{~s}^{-1}$, with FWHM $=0.5,0.8$, and $1.5 \mathrm{~km} \mathrm{~s}^{-1}$, respectively. When needed, we allow a $30 \%$ variation in the FWHM and $0.20 \mathrm{~km} \mathrm{~s}^{-1}$ in $V_{\mathrm{LSR}}$. Some of these small variations may be real, but given the complexity of the line profiles and spatial distribution of the various components, we will not seek a physical interpretation for this. The integrated intensities are summarized in Table 4 , and the physical conditions are derived from these in $\S 4$. 

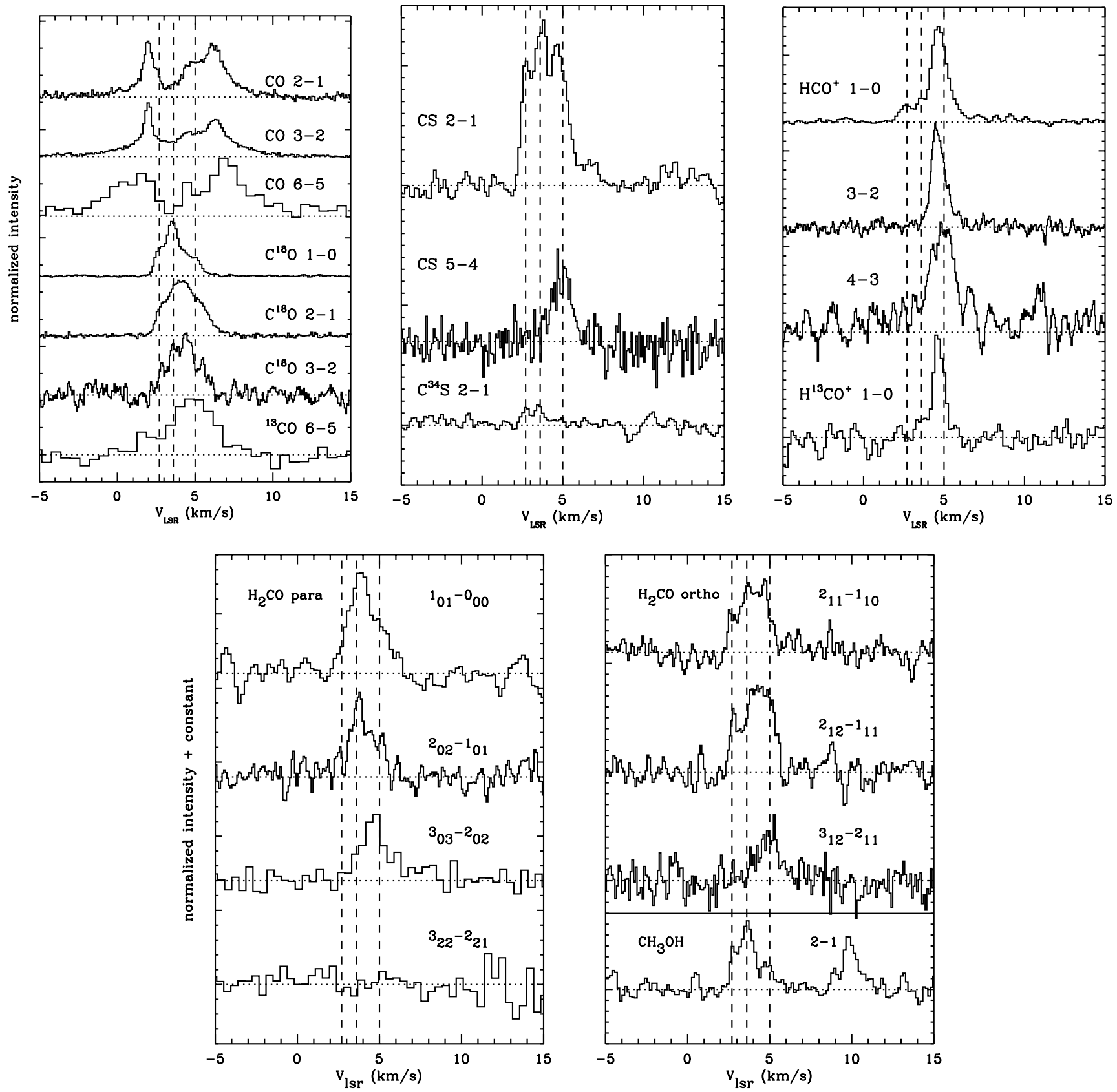

Fig. 3.-Emission lines of $\mathrm{CO}, \mathrm{CS}, \mathrm{HCO}^{+}, \mathrm{H}_{2} \mathrm{CO}$, and $\mathrm{CH}_{3} \mathrm{OH}$ toward Elias 29. The vertical dashed lines indicate different dynamic components at $V_{\mathrm{LSR}}=2.7,3.8$, and $5.0 \mathrm{~km} \mathrm{~s}^{-1}$. The spectra have been arbitrarily scaled and shifted along the intensity axis. See Table 3 for line intensities.

We conclude that when studying individual protostars in the $\rho$ Oph cloud, it must be realized that many different physical components are present within single-dish beams of $\sim 15^{\prime \prime}$ or larger. The spatial information of interferometers is essential here.

\subsection{Small-Scale Emission: Interferometer Maps}

In the OVRO interferometer maps, bright ${ }^{13} \mathrm{CO}$ and $\mathrm{HCO}^{+} 1-0$ emission is detected in the direct neighborhood of Elias 29 (Fig. 4). The ${ }^{13} \mathrm{CO}$ emission is most strongly peaked on the infrared position, and with a spatial FWHM $\sim 6^{\prime \prime}$ (900 AU), it is resolved along the east-west direction, where the OVRO beam is smallest $\left(3^{\prime \prime}\right)$. This is the
Elias 29 core, which likely consists of a disk/envelope system (§ 4.2). An extension of $\sim 12^{\prime \prime}$ toward the southwest is visible in both lines, but most prominently in $\mathrm{HCO}^{+} 1-0$, which must be attributed to the ridge discussed above. Indeed, on this small scale the $\mathrm{HCO}^{+}$emission is also parallel to the IRAM $30 \mathrm{~m} 1.3 \mathrm{~mm}$ continuum emission (Fig. 4).

The OVRO ${ }^{13} \mathrm{CO}$ and $\mathrm{HCO}^{+} 1-0$ spectra peak between $V_{\mathrm{LSR}}=5$ and $7 \mathrm{~km} \mathrm{~s}^{-1}$ and show no evidence for the strong, extended 2.7 and $3.8 \mathrm{~km} \mathrm{~s}^{-1}$ foreground emission seen in the single-dish maps (Fig. 5). The $\mathrm{C}^{18} \mathrm{O} 1-0$ emission is spectacularly absent in the interferometer spectrum; it is at least a factor of 10 weaker compared to the single-dish data. After dilution to the NRAO $12 \mathrm{~m}$ beam (taking the emitting area from the OVRO ${ }^{13} \mathrm{CO}$ image), the OVRO $\mathrm{C}^{18} \mathrm{O}$ signal is 

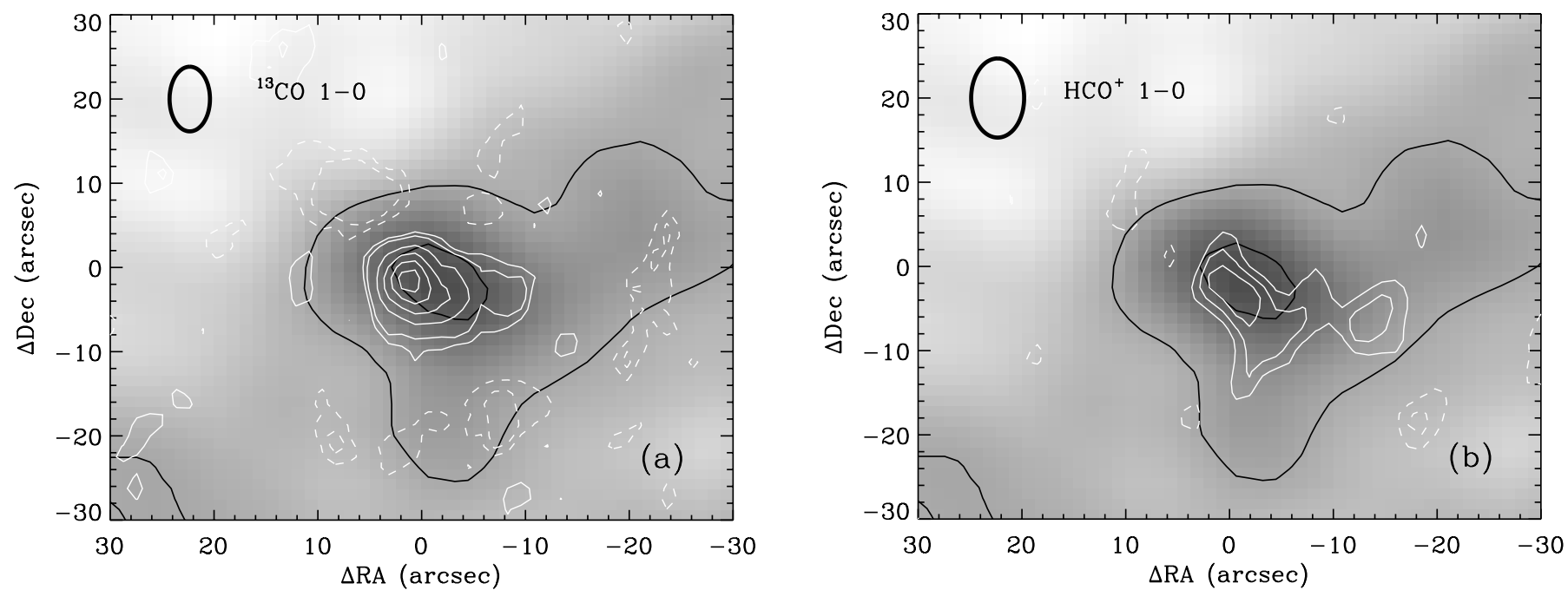

Fig. 4. - Integrated (a) OVRO ${ }^{13} \mathrm{CO} 1-0$ and $(b) \mathrm{HCO}^{+} 1-0$ emission in the Elias 29 region (white contours) superposed on the $1.3 \mathrm{~mm}$ continuum map (gray scale and black contours). Contour levels are drawn at $-3,-2,2,3,6, \ldots, 15$ and $-3,-2,2,3$, and 4 times the noise level $\left(0.48\right.$ and 0.90 Jy beam ${ }^{-1}$ for ${ }^{13} \mathrm{CO} 1-0$ and $\mathrm{HCO}^{+} 1-0$, respectively). The contours for negative signals are given by white dashed lines. Contour levels for the $1.3 \mathrm{~mm}$ map are the same as in Fig. 1 . The synthesized OVRO beam size is given in the top left-hand corners.

even a factor of 60 weaker than the bright detection with the NRAO $12 \mathrm{~m}$ telescope. This shows again that the bulk of the molecular material in the Elias 29 line of sight is not associated with Elias 29 but instead is present in extended foreground clouds, which are resolved out by the OVRO interferometer.

It is interesting to note that the OVRO line profiles are double peaked (Fig. 5), with a $5.0 \mathrm{~km} \mathrm{~s}^{-1}$ component

TABLE 4

Integrated Intensities of Decomposed Lines at Center Position

\begin{tabular}{|c|c|c|c|c|}
\hline \multirow[b]{2}{*}{ Molecule } & \multirow[b]{2}{*}{ Transition } & \multicolumn{3}{|c|}{$\int T_{\mathrm{MB}} d v$ FOR Velocity COMPONENT ${ }^{\mathrm{a}}$} \\
\hline & & $2.7 \mathrm{~km} \mathrm{~s}^{-1}$ & $3.8 \mathrm{~km} \mathrm{~s}^{-1}$ & $5.0 \mathrm{~km} \mathrm{~s}^{-1}$ \\
\hline \multirow{3}{*}{$\mathrm{C}^{18} \mathrm{O}$} & $1-0$ & 1.78 & 5.18 & 3.97 \\
\hline & $2-1$ & 1.93 & 4.19 & 9.50 \\
\hline & $3-2$ & 1.00 & 2.75 & 6.78 \\
\hline $\mathrm{C}^{17} \mathrm{O}^{\mathrm{b}} \ldots \ldots \ldots$ & $1-0$ & 0.66 & 1.94 & $<1.6$ \\
\hline \multirow[t]{3}{*}{$\mathrm{CS} \ldots \ldots \ldots \ldots$} & $2-1$ & 0.69 & 1.01 & 1.72 \\
\hline & $5-4$ & $<0.07$ & $<0.09$ & 0.82 \\
\hline & $7-6$ & $<0.13$ & $<0.13$ & $<0.13$ \\
\hline $\mathrm{C}^{34} \mathrm{~S} \ldots \ldots \ldots \ldots$ & $2-1$ & 0.09 & 0.12 & $<0.05$ \\
\hline \multirow[t]{4}{*}{$\mathrm{p}-\mathrm{H}_{2} \mathrm{CO} \ldots \ldots$} & $1_{01}-0_{00}$ & $<0.15$ & 0.55 & 0.65 \\
\hline & $2_{02}-1_{01}$ & $<0.07$ & 0.43 & 0.39 \\
\hline & $3_{03}-2_{02}$ & $<0.03$ & $<0.04$ & 0.82 \\
\hline & $3_{22}-2_{21}$ & $<0.03$ & $<0.04$ & $<0.12$ \\
\hline \multirow[t]{3}{*}{$\mathrm{o}-\mathrm{H}_{2} \mathrm{CO} \ldots \ldots$} & $2{ }_{12}-1_{11}$ & 0.30 & 0.50 & 1.03 \\
\hline & $2_{11}-1_{10}$ & 0.21 & 0.52 & 0.65 \\
\hline & $3_{12}-2_{11}$ & $<0.06$ & $<0.09$ & 0.78 \\
\hline \multirow[t]{3}{*}{$\mathrm{CH}_{3} \mathrm{OH} \ldots \ldots$} & $2-196739.4$ & 0.03 & 0.14 & $<0.05$ \\
\hline & 2-196741.4 & 0.06 & 0.18 & 0.10 \\
\hline & $2-196744.6$ & $<0.02$ & $<0.02$ & $<0.05$ \\
\hline \multirow[t]{3}{*}{$\mathrm{HCO}^{+} \ldots \ldots .}$. & $1-0$ & 0.49 & 0.46 & 4.26 \\
\hline & $3-2$ & $<0.04$ & $<0.07$ & 3.17 \\
\hline & 4-3 & $<0.05$ & $<0.08$ & 0.76 \\
\hline $\mathrm{H}^{13} \mathrm{CO}^{+} \ldots \ldots$ & $1-0$ & $<0.02$ & 0.05 & 0.33 \\
\hline
\end{tabular}

a Relative uncertainties are smaller than the error in the absolute calibration $(25 \%)$.

b Derived from 112,360.0 MHz fine structure line, multiplied by a factor of 3 to correct for emission in other fine structure lines. emitting in the dense ridge (Fig. 6). Emission at the central infrared position is strongest at $6 \mathrm{~km} \mathrm{~s}^{-1}$. Whether this is a true dynamical difference between the Elias 29 core and the ridge or artificially created by self-absorption at $\sim 5.5 \mathrm{~km} \mathrm{~s}^{-1}$ is difficult to answer at present. In this paper we will keep referring to this as the $5.0 \mathrm{~km} \mathrm{~s}^{-1}$ component. A disk/envelope/ridge decomposition is attempted in $\S 4.2$.

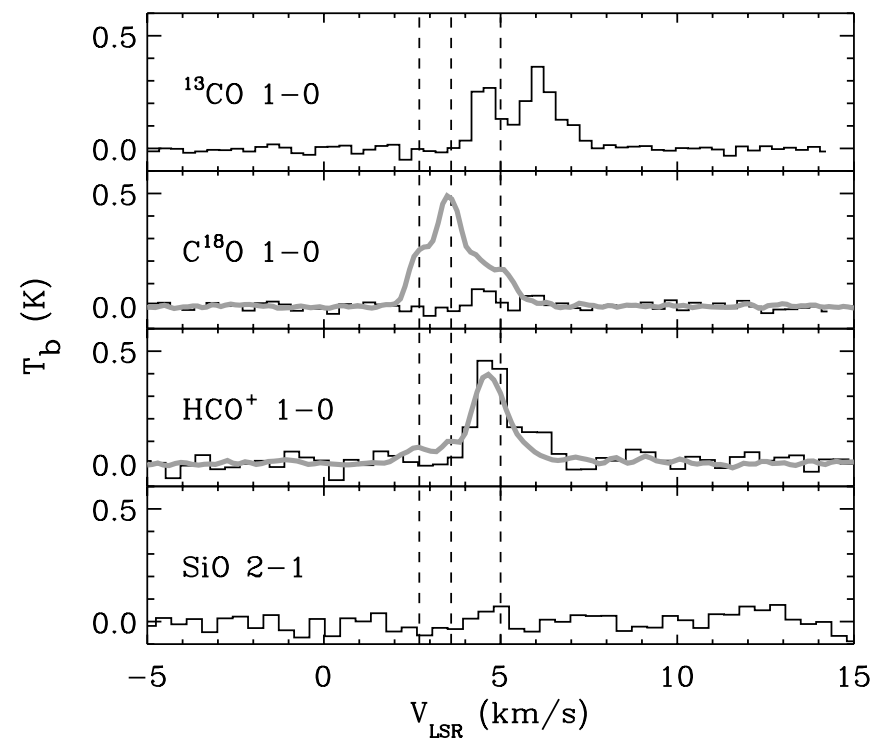

FIG. 5.-Average line profiles of the central region with more than $2 \sigma$ emission in the OVRO maps, which is an area of $380 \operatorname{arcsec}^{2}$ for ${ }^{13} \mathrm{CO}$ and $650 \operatorname{arcsec}^{2}$ for $\mathrm{HCO}^{+}$. For the nondetected $\mathrm{C}^{18} \mathrm{O}$ and $\mathrm{SiO}$ lines, we took the central $400 \operatorname{arcsec}^{2}$. The brightness temperature is correspondingly diluted by factors of 7.4, 5.9, 5.8, and 6.4 from top to bottom to derive the expected signal in the single-dish NRAO $12 \mathrm{~m}$ beam. The smooth gray line for $\mathrm{C}^{18} \mathrm{O}$ and $\mathrm{HCO}^{+}$is the NRAO $12 \mathrm{~m}$ single-dish emission divided by factors of 13 and 9, respectively (for clarity). The vertical dashed lines represent the single-dish emission-line components shown in Fig. 3. 

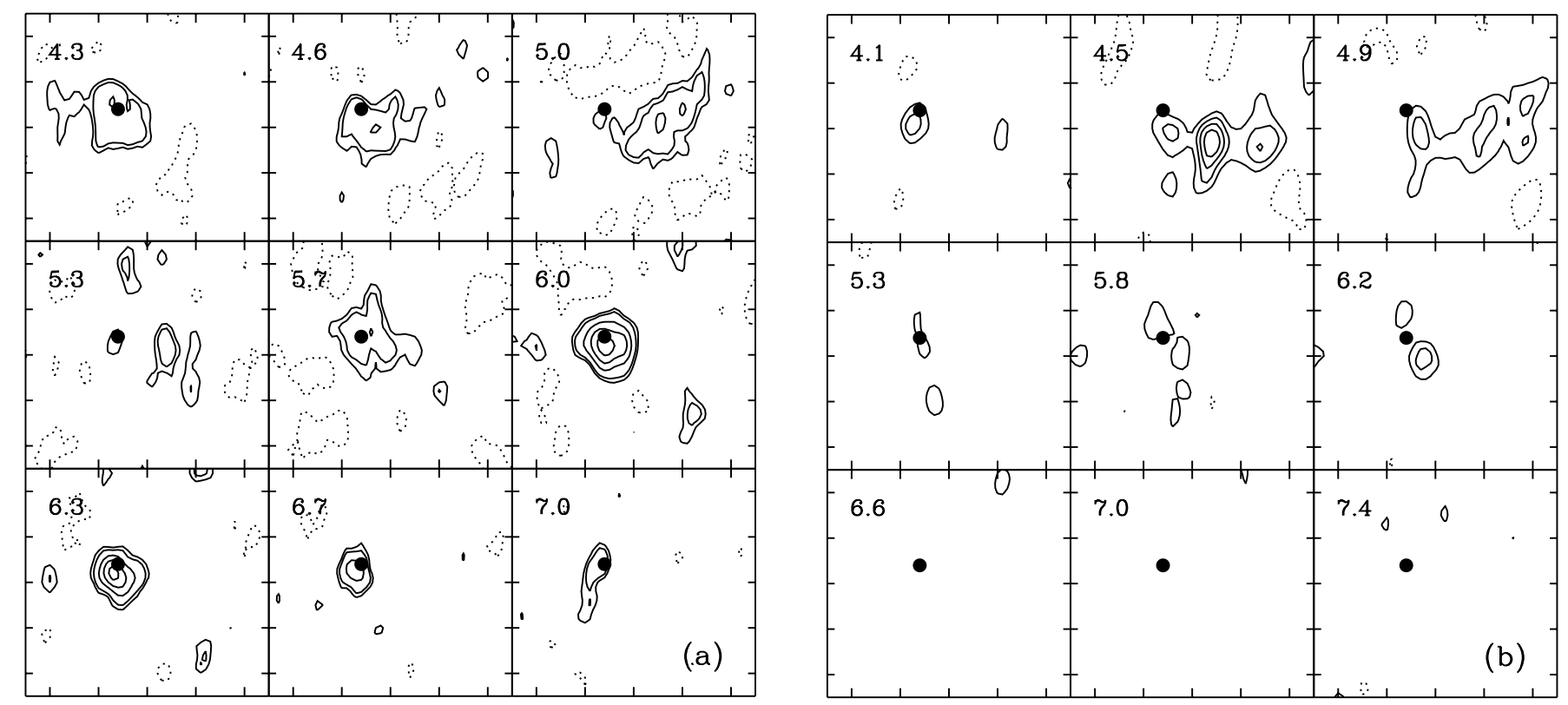

FIG. 6. - Channel maps of $(a)$ OVRO ${ }^{13} \mathrm{CO} 1-0$ and $(b) \mathrm{HCO}^{+} 1-0$ emission in the Elias 29 region. The map size is $50^{\prime \prime}$. The bullets indicate the map centers and facilitate comparison of the panels. Contour levels are drawn at $-2,2,3,6,9$, and $12\left({ }^{13} \mathrm{CO}\right)$ and $-2,2,3,4$, and 5 times the noise level $(0.13$ and $0.26 \mathrm{Jy}$ beam $^{-1}$, respectively), with the negative contour indicated with dots. Extended emission toward the west is present at velocities near $5.0 \mathrm{~km} \mathrm{~s}^{-1}$ in both lines and coincides with the large-scale dense ridge seen in our $\mathrm{HCO}^{+} 3-2$ single-dish map.

\section{DISCUSSION: PHYSICAL CONDITIONS}

It is our aim to derive the physical structure of the surroundings of Elias 29 and to locate the origin of the ices, seen abundantly along this line of sight (Boogert et al. 2000b). In $\S 4.1$ the extended clouds at 2.7 and $3.8 \mathrm{~km} \mathrm{~s}^{-1}$ identified above are discussed; $\S \S 4.2$ and 4.3 describe the more immediate circumstellar environment of Elias 29 in terms of a near face-on disk, a remnant envelope, and the dense ridge from which the star may have formed (see Fig. 7). The physical conditions in each of the components are constrained using the intensities and intensity ratios of

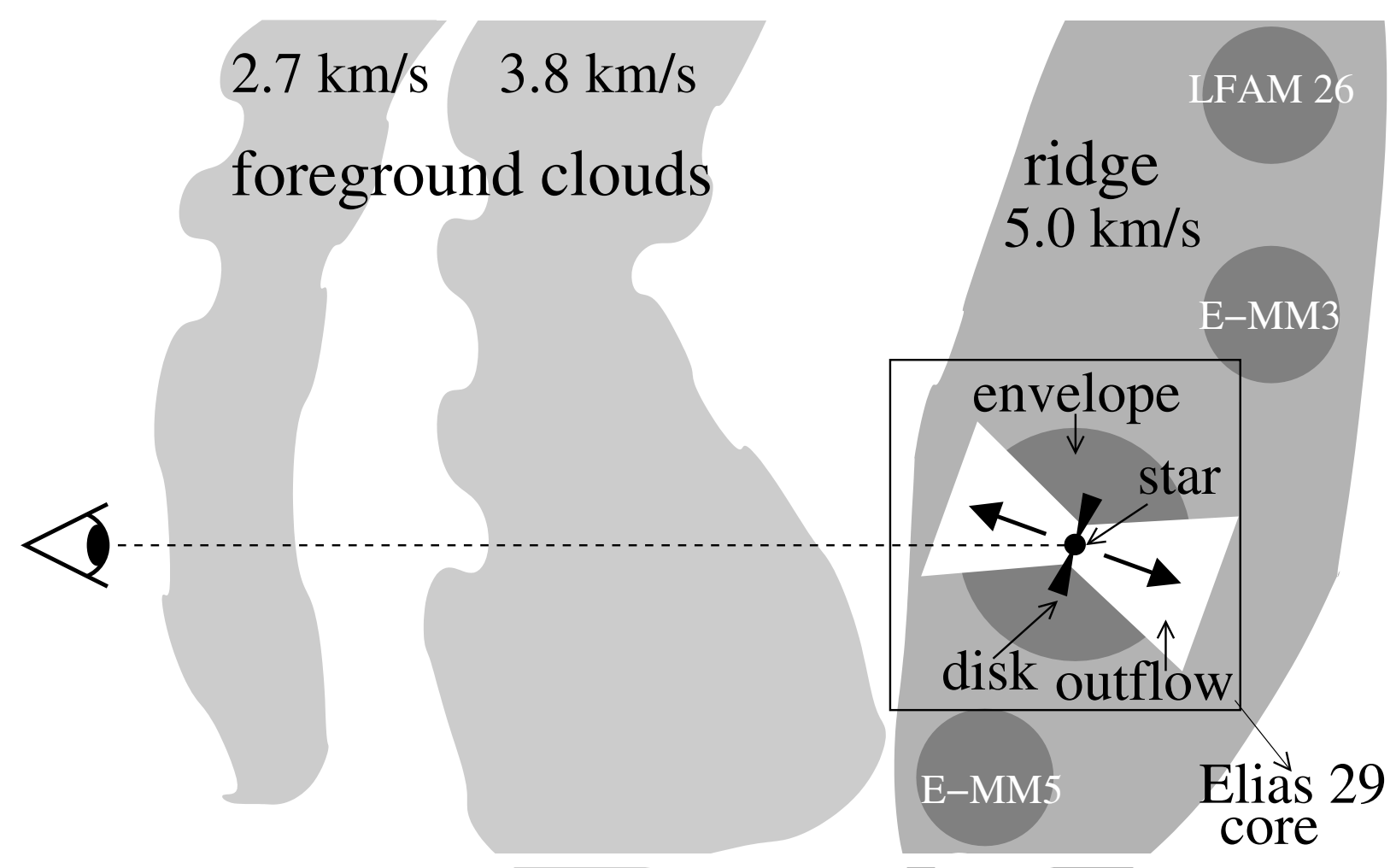

FIG. 7.-Schematic overview of the proposed geometry of Elias 29 and its environment (not to scale). The line of sight to Elias 29 may cross part of the envelope if the system is slightly more inclined than shown here. Most of the ice absorption bands originate in the foreground clouds. 
the single-dish and interferometer line emission, the $1.3 \mathrm{~mm}$ continuum emission (Motte et al. 1998), and the infrared SED (Boogert et al. 2000b). This is linked to the ice observations in $\S 4.4$.

\subsection{Foreground Clouds}

The $\mathrm{C}^{18} \mathrm{O}$ line emission at 2.7 and $3.8 \mathrm{~km} \mathrm{~s}^{-1}$ is extended over several arcminutes (Fig. 2) and thus is associated with the overall $\rho$ Oph cloud complex. Assuming that the clouds are sufficiently homogeneous, we can use the ratios of the decomposed line intensities (Table 4) to find the density and temperature, and subsequently the column density, in each of the clouds. We use the escape probability method described by Jansen (1995) to calculate the molecular excitation.

The most useful constraints on the density and temperature are given by the intensity ratios of $\mathrm{C}^{18} \mathrm{O} 1-0 / 3-2$ and $\mathrm{HCO}^{+} 1-0 / 3-2$. Figure 8 visualizes the density and temperature values allowed by the observed ratios, taking into account line opacity effects. The $2.7 \mathrm{~km} \mathrm{~s}^{-1}$ component has a temperature $T=15 \pm 5 \quad \mathrm{~K}$ and a density $n\left(\mathrm{H}_{2}\right)=(1 \pm 0.5) \times 10^{4} \mathrm{~cm}^{-3}$. For the $3.8 \mathrm{~km} \mathrm{~s}^{-1}$ component, the density is less than $10^{5} \mathrm{~cm}^{-3}$, but the temperature cannot be significantly constrained from the line ratios. Here we use the far-infrared SED to find that $T_{\text {kin }}=25 \pm 15 \mathrm{~K}(\S 4.2)$. Taking a $\mathrm{C}^{18} \mathrm{O}$ abundance with respect to $\mathrm{H}_{2}$ of $3.6 \times 10^{-7}\left[N(\mathrm{CO}) / N\left(\mathrm{C}^{18} \mathrm{O}\right)=560\right.$; Wilson \& Rood 1994; $N\left(\mathrm{H}_{2}\right) / N(\mathrm{CO})=5000$; Lacy et al. 1994], the $\mathrm{H}_{2}$ column densities of the 2.7 and $3.8 \mathrm{~km} \mathrm{~s}^{-1}$ clouds are $5 \times 10^{21}$ and $1.4 \times 10^{22} \mathrm{~cm}^{-2}$, respectively (Table 5). This also assumes that $\mathrm{CO}$ is not strongly depleted in these

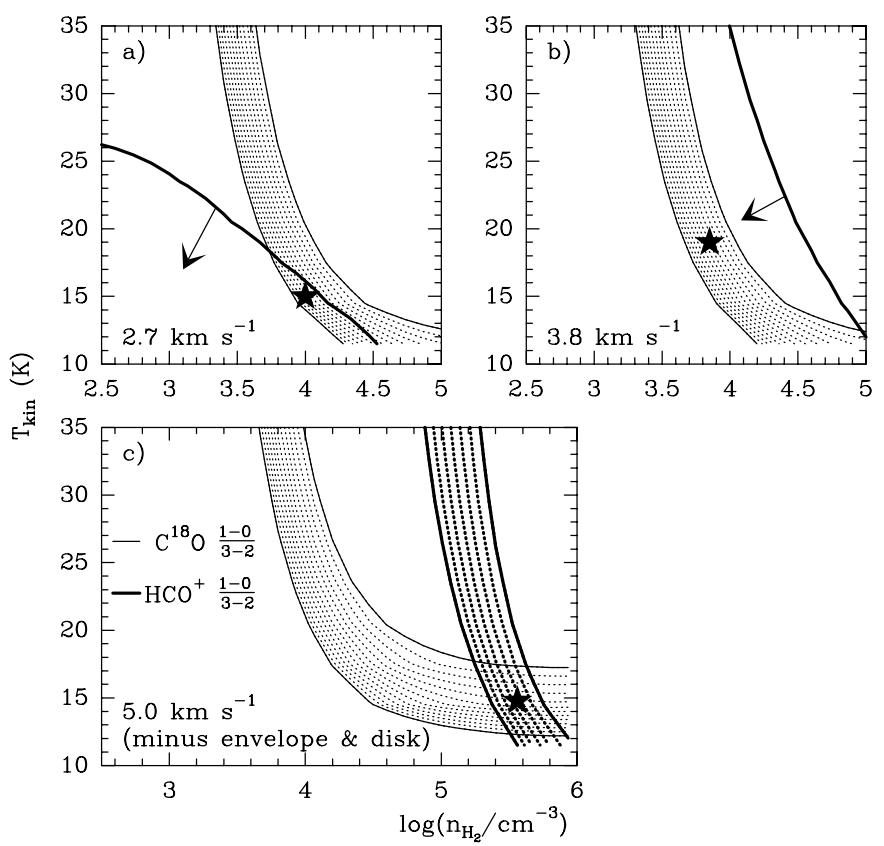

FIG. 8.-Temperature and density range constrained by intensity ratios of $\mathrm{C}^{18} \mathrm{O} 1-0 / 3-2$ (thin lines) and $\mathrm{HCO}^{+} 1-0 / 3-2$ (heavy lines) for the foreground clouds at $(a) V_{\mathrm{LSR}}=2.7$ and $(b) 3.8 \mathrm{~km} \mathrm{~s}^{-1}$ and for the ridge material at $(c) 5.0 \mathrm{~km} \mathrm{~s}^{-1}$. The intensity ratios for the ridge material have been corrected for the expected contribution from the envelope and disk of Elias 29. The filled star indicates the adopted temperature and density for each of the components. clouds, which is validated in $\S 4.5$. These parameters fit the optically thin $\mathrm{C}^{17} \mathrm{O}$ lines also, indicating that a correct line opacity was used

With these physical parameters at hand, we calculate that the optical depth of the clouds in the CO 5-6 transition is $\sim 4$. This is sufficiently high to explain the absorption in the CO 6-5 lines at 2.7 and $3.8 \mathrm{~km} \mathrm{~s}^{-1}$ (Fig. 3). The bright $\mathrm{CO}$ 6-5 emission is closely associated with Elias 29 (C. Ceccarelli et al. 2002, in preparation), and therefore the 2.7 and 3.8 $\mathrm{km} \mathrm{s}^{-1}$ clouds are located in front of this source.

Independent information on the physical conditions in these extended foreground clouds is obtained from the SED longward of $100 \mu \mathrm{m}(\S 4.2)$. The columns and temperatures also compare favorably with the extended cold dust found by sensitive $180-1100 \mu \mathrm{m}$ balloon-based measurements (Ristorcelli et al. 1999; Table 6).

The observed lines of CS, $\mathrm{H}_{2} \mathrm{CO}$, and $\mathrm{CH}_{3} \mathrm{OH}$ do not further constrain the physical conditions, but they can be used to derive abundances. These are listed in Table 5 and further discussed in $\S 4.5$.

\subsection{The Circumstellar Environment of Elias 29}

Understanding the circumstellar environment of Elias 29 requires the combination of several pieces of crucial information: the single-dish $\mathrm{HCO}^{+} 3-2$ map and $1.3 \mathrm{~mm}$ dustcontinuum distribution (Fig. 1), the spatially resolved emission in ${ }^{13} \mathrm{CO} 1-0$ and $\mathrm{HCO}^{+} 1-0$ observed by OVRO (Fig. 4 ), and the infrared SED obtained by the SWS and LWS spectrometers of the ISO satellite (Boogert et al. 2000b; Fig. 9).

The $\mathrm{HCO}^{+} 3-2$ and $1.3 \mathrm{~mm}$ continuum maps show that Elias 29 is located in a narrow, dense ridge, $\lesssim 30^{\prime \prime}$ wide and several arcminutes long. The strongest $\mathrm{HCO}^{+}$ 3-2 peak in the ridge is situated near Elias 29. The OVRO images and channel maps (Figs. 4 and 6) show that part of the ${ }^{13} \mathrm{CO}$ and $\mathrm{HCO}^{+}$emission is centered on Elias 29, while a significant fraction follows the crest of the ridge, $10^{\prime \prime}-20^{\prime \prime}$ offset to the west/southwest. This shows that multiple components are present in the immediate vicinity of Elias 29 (i.e., within a typical single-dish beam) in addition to the multiple velocity components along the line of sight ( $\S 4.1)$. The velocity coincidence between the ridge and Elias 29 further supports that the star formed from the ridge.

\subsubsection{SED and Continuum Modeling}

The most important clues about the nature of Elias 29 are offered by the infrared SED. Because of the large beam with which these measurements were taken $(\S 2)$, the SED reflects not only Elias 29 but also many of the other components identified above. Our ability to derive accurate parameters for each component therefore proved essential in helping to understand the nature of Elias 29. The SED (Fig. 9) is remarkably flat between 10 and $200 \mu \mathrm{m}$. At $10 \mu \mathrm{m}$ there is a prominent silicate absorption band, and at $5 \mu \mathrm{m}$ the SED shows a broad peak, on top of which a number of sharp ice absorption features are present. Below $2 \mu \mathrm{m}$ the emission drops off sharply. Flat protostellar SEDs are generally explained by a circumstellar disk. Compared to other circumstellar material distributions, a disk, especially a flared one, has the surface area versus temperature distribution required to create a flat $S E D$. 
TABLE 5

Physical Conditions in Elias 29 Foreground Clouds and Ridge and Comparison with Other Clouds

\begin{tabular}{|c|c|c|c|c|c|c|}
\hline \multirow[b]{2}{*}{ QUANTITY } & \multicolumn{3}{|c|}{ Cloud Component } & \multirow[b]{2}{*}{$\mathrm{TMC} 1^{\mathrm{a}}$} & \multicolumn{2}{|c|}{ NGC $1333^{b}$} \\
\hline & $2.7 \mathrm{~km} \mathrm{~s}^{-1}$ & $3.8 \mathrm{~km} \mathrm{~s}^{-1}$ & $5.0 \mathrm{~km} \mathrm{~s}^{-1}$ & & IRAS 4A & IRAS 4B \\
\hline$b_{\mathrm{D}}\left(\mathrm{km} \mathrm{s}^{-1}\right) \ldots \ldots \ldots \ldots \ldots \ldots$ & 0.3 & 0.5 & 1.0 & $\ldots$ & $\ldots$ & $\ldots$ \\
\hline$T_{\text {kin }}(\mathrm{K}) \ldots \ldots \ldots \ldots \ldots \ldots \ldots$ & $15(5)$ & $25(15)$ & $15(5)$ & $\ldots$ & $\ldots$ & $\ldots$ \\
\hline$n\left(\mathrm{H}_{2}\right)\left(\times 10^{4} \mathrm{~cm}^{-3}\right) \ldots \ldots \ldots$ & $1.0(0.5)$ & $<10$ & $40(20)$ & $\ldots$ & $\ldots$ & $\ldots$ \\
\hline$N\left(\mathrm{H}_{2}\right)\left(\times 10^{22} \mathrm{~cm}^{-2}\right) \ldots \ldots$. & $0.5(0.2)$ & $1.4(0.2)$ & $1.0(0.2)$ & 1.0 & 14 & 6 \\
\hline$X\left(\mathrm{C}^{18} \mathrm{O}\right)^{\mathrm{c}}\left(\times 10^{-9}\right) \ldots \ldots \ldots$ & 360 & 360 & 360 & 304 & 40 & 70 \\
\hline$X\left(\mathrm{C}^{17} \mathrm{O}\right)^{\mathrm{c}}\left(\times 10^{-9}\right) \ldots \ldots \ldots$ & 110 & 110 & 110 & 95 & 7.6 & 19 \\
\hline$X(\mathrm{CS})\left(\times 10^{-9}\right) \ldots \ldots \ldots \ldots \ldots$ & 6.0 & 15.0 & $\ldots^{d}$ & 6 & 1.2 & 0.2 \\
\hline$X\left(\mathrm{HCO}^{+}\right)\left(\times 10^{-9}\right) \ldots \ldots \ldots$ & 0.6 & 0.7 &.$^{\mathrm{d}}$ & 9 & 0.4 & 0.1 \\
\hline$X\left(\mathrm{o}-\mathrm{H}_{2} \mathrm{CO}\right)\left(\times 10^{-9}\right) \ldots \ldots$ & 3.0 & 8.0 &.$^{\mathrm{d}}$ & 50 & 0.4 & 0.9 \\
\hline$X\left(\mathrm{p}-\mathrm{H}_{2} \mathrm{CO}\right)\left(\times 10^{-9}\right) \ldots \ldots$ & $<1.0$ & 2.7 & $\ldots^{\mathrm{d}}$ & $\ldots$ & $\ldots$ & $\ldots$ \\
\hline$X\left(\mathrm{CH}_{3} \mathrm{OH}\right)\left(\times 10^{-9}\right) \ldots \ldots$ & 0.4 & 0.5 & $\ldots^{\mathrm{d}}$ & 3 & $\ldots$ & $\ldots$ \\
\hline$A_{V}(\mathrm{mag}) \ldots \ldots \ldots \ldots \ldots \ldots$ & 2.9 & 8.2 & $<5.9^{\mathrm{e}}$ & $\ldots$ & $\ldots$ & $\ldots$ \\
\hline
\end{tabular}

NotE.-All abundances $X$ are with respect to $\mathrm{H}_{2}$ and have an error of $50 \%$.

a On "CP" peak; Pratap et al. 1997; Ohishi \& Kaifu 1998.

b Blake et al. 1995, assuming $N\left(\mathrm{H}_{2}\right)$ from dust.

${ }^{c}$ From $\mathrm{H}_{2}$ using standard relations (see text), except TMC 1 .

${ }^{\mathrm{d}}$ Line intensities in the ridge have to be corrected for expected contribution from envelope and disk (see text). No independent determination of abundances is therefore possible.

e Upper limit; only a fraction of the ridge material may actually be in front of Elias 29.

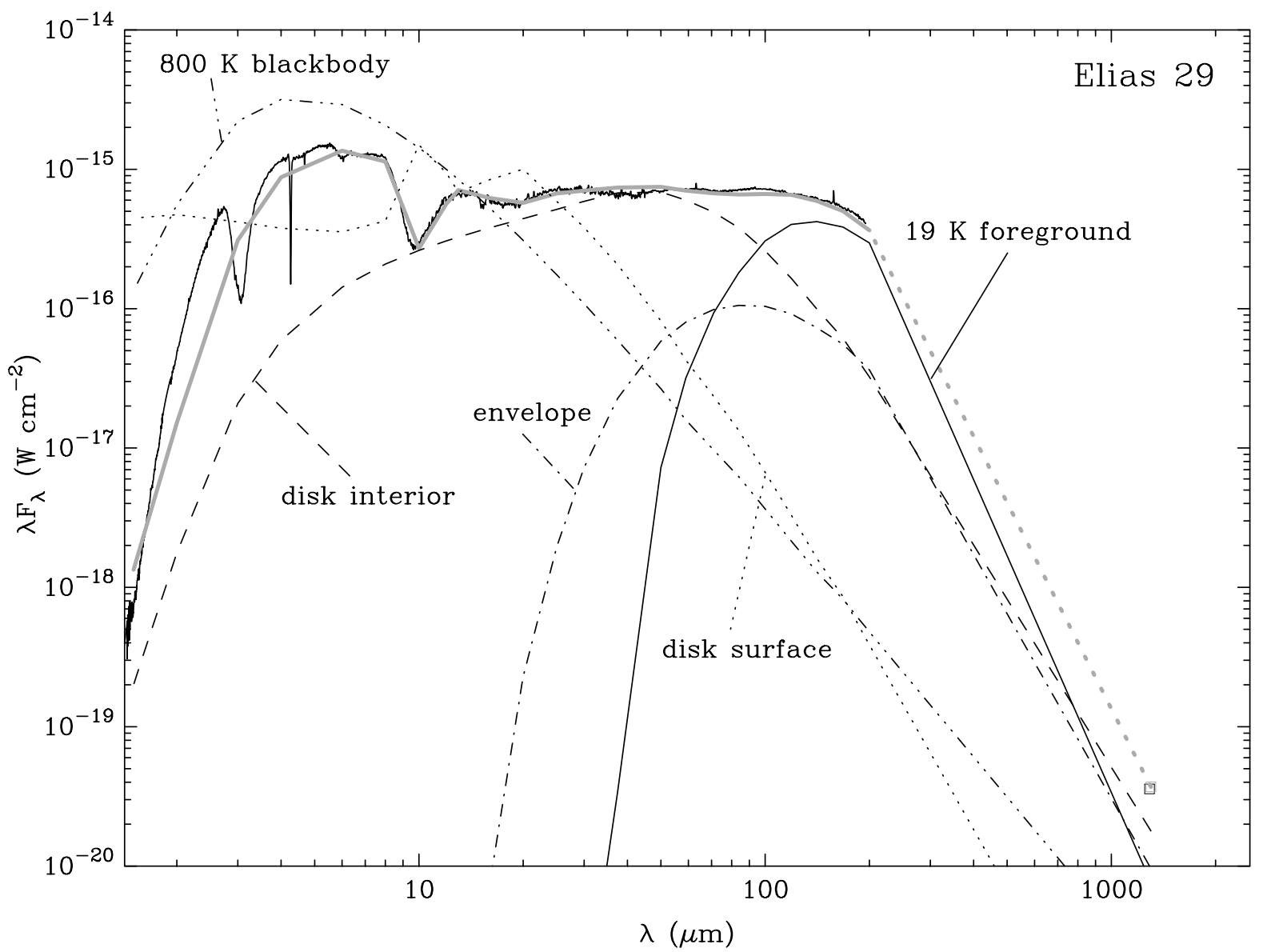

FIG. 9.--Infrared SED of Elias 29 as observed by ISO LWS and SWS (thin line with ice and silicate absorption features; Boogert et al. 2000b) combined with the $1.3 \mathrm{~mm}$ flux in the $15^{\prime \prime}$ IRAM $30 \mathrm{~m}$ beam (open square; Motte et al. 1998). The thick gray line shows the modeled SED as described in $\S 4.2$. It consists of four components: (1) a $0.012 M_{\odot}$ flared circumstellar disk following Chiang \& Goldreich (1997) with a two-layer vertical temperature structure (cool interior [long-dashed line] and superheated surface [dotted line]); (2) a $0.12 M_{\odot}$ collapsing envelope (dot-dashed line); (3) an ad hoc $800 \mathrm{~K}$ central blackbody with a $1.4 \mathrm{AU}$ radius (triple-dot-dashed line), required to fit the flux maximum at $\lambda \sim 5 \mu \mathrm{m}$ and probably corresponding to the warmest regions of the disk close to the star and/or the stellar photosphere extincted by the disk; and (4) a $19 \mathrm{~K}$ foreground layer (thin solid line, peaking at $\sim 120 \mu \mathrm{m}$ ) with a column density of $N\left(\mathrm{H}_{2}\right)=1.9 \times 10^{22} \mathrm{~cm}^{-2}$, as derived for the material at $V_{\mathrm{LSR}}=2.7$ and $3.8 \mathrm{~km} \mathrm{~s}^{-1}$ in $\S 4.1$, which is responsible for the continuum absorption below $3 \mu \mathrm{m}$ in the final four-component model and for the prominent silicate and ice absorption bands. 
TABLE 6

Column Density of Cold Gas Derived by Different Methods

\begin{tabular}{|c|c|c|c|}
\hline Method & Component & $\begin{array}{c}N\left(\mathrm{H}_{2}\right)^{\mathrm{a}} \\
\left(\times 10^{22} \mathrm{~cm}^{-2}\right)\end{array}$ & $A_{V}^{\mathrm{b}}$ \\
\hline Silicate absorption ${ }^{\mathrm{c}} \ldots \ldots \ldots$. & & $2.5-6$ & $14-34$ \\
\hline & $b_{D}=1.0 \mathrm{~km} \mathrm{~s}^{-1}$ & $3(0.5)$ & $17(3)$ \\
\hline $\begin{array}{l}4.7 \mu \mathrm{m}^{13} \mathrm{CO} \\
\quad \text { absorption }{ }^{\mathrm{d}} \ldots \ldots \ldots \ldots \ldots \ldots . . . . . . .\end{array}$ & $b_{D}=0.7 \mathrm{~km} \mathrm{~s}^{-1}$ & $11(4)$ & $63(23)$ \\
\hline $\mathrm{C}^{18} \mathrm{O} 2-1$ emission............. & $\begin{array}{l}V_{\mathrm{LSR}}=2.7 \\
V_{\mathrm{LSR}}=3.8 \\
V_{\mathrm{LSR}}=5.0\end{array}$ & $\begin{array}{l}0.5(0.2) \\
1.4(0.2) \\
1.0(0.2)\end{array}$ & $\begin{array}{l}2.9(1.1) \\
8.0(1.1) \\
5.7(1.1)\end{array}$ \\
\hline ISO SED and & & & \\
\hline $\begin{array}{l}\text { IRAM } 30 \mathrm{~m} 1.3 \mathrm{~mm} \ldots . . . \\
0.18-1.1 \mathrm{~mm}\end{array}$ & Envelope & $0.3-1$ & $1.7-6$ \\
\hline 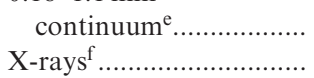 & $10-15 \mathrm{~K}$ & $\begin{array}{c}0.5-5 \\
3.8\end{array}$ & $\begin{array}{l}2.9-29 \\
22\end{array}$ \\
\hline
\end{tabular}

a Assuming $N\left(\mathrm{H}_{2}\right) / N(\mathrm{CO})=5 \times 10^{3}$.

${ }^{\mathrm{b}}$ Conversion factor $A_{\mathrm{V}}=N\left(\mathrm{H}_{2}\right) \times 8.6 / 15 \times 10^{21}$; Bohlin, Savage, \& Drake 1978

c See Boogert et al. 2000b.

d A. C. A. Boogert et al. 2002, in preparation.

e Balloon experiment; Ristorcelli et al. 1999.

${ }^{\mathrm{f}}$ Imanishi, Koyama, \& Tsuboi 2001

For our analysis, we adopt the flared disk model of Chiang \& Goldreich (1997) with the parameters listed in Table 7. The flatness of the SED is explained in this model by a superheated surface layer. The continuum emission of the disk is modeled using the radiative transfer part of the Monte Carlo code of Hogerheijde \& van der Tak (2000); this part of the code simply calculates the expected continuum emission from a given distribution of density and temperature by building up a grid of points on the sky, for each of which the radiative transfer is solved along straight lines. The resulting grid is then convolved with the appropriate beam sizes. Because of the large number of parameters involved, their values should be considered as representative only. We believe, however, that the general characteristics of the model are firmly established. The flatness of the SED over a large wavelength range (4-100 $\mu \mathrm{m}$; Fig. 9) limits the inclination of the system to less than $60^{\circ}\left(90^{\circ}\right.$ being edge-on; Chiang \& Goldreich 1999). Such a low inclination cannot explain the deep ice and silicate absorption features that are visible in the observed SED; these must originate in the envelope and foreground clouds. The low disk inclination is compatible with the very high velocity $\left(\sim 80 \mathrm{~km} \mathrm{~s}^{-1}\right)$, variable outflowing hot $\mathrm{CO}$ gas seen in this object (A. C. A. Boogert, G. A. Blake, \& M. R. Hogerheijde 2002, in preparation). A fully face-on orientation is, however, not likely given the presence of low surface brightness scattered $K$ band light out to distances of $15^{\prime \prime}$ (2400 AU) from the central object (Zinnecker, Perrier, \& Chelli 1988). This light presumably traces the outflow lobes, adjacent to the remnant envelope and the outer edges of the disk. The emission is extended along the southwest/northeast direction, perpendicular to the high-velocity outflowing gas (C. Ceccarelli et al. 2002, in preparation) with an axis ratio compatible with an inclination of $\sim 30^{\circ}$. Flattening on a similar scale is observed in the OVRO images and in the $1.3 \mathrm{~mm}$ continuum IRAM $30 \mathrm{~m}$ single-dish map.

The flux between 20 and $40 \mu \mathrm{m}$ indicates a temperature scaled upward by a factor of 2.25 with respect to the values used by Chiang \& Goldreich (1997). The central star is
TABLE 7

Parameters of Disk and Envelope Model

\begin{tabular}{|c|c|}
\hline Item & Value \\
\hline \multicolumn{2}{|c|}{ Disk: Chiang \& Goldreich 1997} \\
\hline 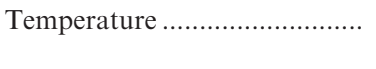 & $\begin{array}{l}2.25 \text { times standard model } \\
\text { of Chiang \& Goldreich }\end{array}$ \\
\hline 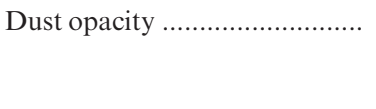 & $\begin{array}{l}\text { Ossenkopf \& Henning 1994; } \\
\text { MRN model with } 10^{6} \mathrm{yr} \text { of } \\
\text { coagulation at } 10^{5} \mathrm{~cm}^{-3}\end{array}$ \\
\hline Inner radius (AU). & 0.01 \\
\hline Outer radius (AU).. & 500 \\
\hline $\operatorname{Mass}\left(M_{\odot}\right) \ldots \ldots \ldots \ldots \ldots$ & 0.012 \\
\hline $\begin{array}{l}\text { Mass in superheated } \\
\text { surface layer }(\%)\end{array}$ & 2.5 \\
\hline
\end{tabular}

Envelope: Shu 1977

\begin{tabular}{|c|c|}
\hline Sound speed $\left(\mathrm{km} \mathrm{s}^{-1}\right) \ldots \ldots \ldots \ldots \ldots$ & 0.13 \\
\hline 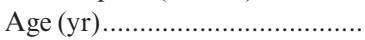 & $2.2 \times 10^{5}$ \\
\hline $\begin{array}{l}\text { Radius of collapse expansion } \\
\text { wave (AU) }\end{array}$ & 6000 \\
\hline 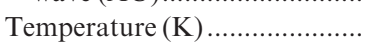 & $35(r / 1000 \mathrm{AU})^{-0.4}$ \\
\hline 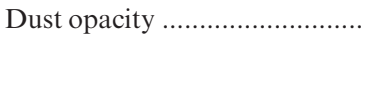 & $\begin{array}{l}\text { Ossenkopf \& Henning } 1994 ; \\
\text { MRN model with } 10^{6} \mathrm{yr} \text { of } \\
\text { coagulation at } 10^{5} \mathrm{~cm}^{-3}\end{array}$ \\
\hline 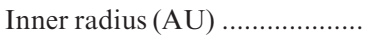 & $500\left(3^{\prime \prime}\right)$ \\
\hline Outer radius (AU) ....................... & $6000\left(39^{\prime \prime}\right)$ \\
\hline $\operatorname{Mass}\left(M_{\odot}\right)$ & 0.12 \\
\hline
\end{tabular}

therefore approximately 50 times brighter than their standard model, presumably because it has a higher mass (using the scaling relation $T \propto L^{1 / 5}$; Chandler \& Richer 2000). The disk midplane dominates the emission beyond $25 \mu \mathrm{m}$, and the shape of the SED at these wavelengths limits the disk radius to $\sim 500 \mathrm{AU}$. The warmer surface layer dominates the shorter wavelengths and generate a silicate emission feature at $10 \mu \mathrm{m}$ (Fig. 9). The disk model can explain only $\sim 20 \%$ of the emission maximum around $5 \mu \mathrm{m}$. Indeed, speckle observations of Elias 29 reveal the presence of a $\sim 400$ AU radius thermally emitting region ( $T$ equals a few $100 \mathrm{~K}$; i.e., not scattered light) responsible for $\sim 20 \%$ of the $M$-band flux (Zinnecker et al. 1988), which in our model, is explained by the warm disk surface layer. The observed remaining $80 \%$ of the $M$-band emission originates from hot dust within a few AU from the central object. Perhaps this is a puffed-up hot rim at the inner disk edge, just outside the region where dust has evaporated $\left(T_{\text {subl }} \sim 1500 \mathrm{~K}\right.$; Dullemond, Dominik, \& Natta 2001; Zinnecker et al. 1988). The innermost, hottest part of the rim may be related to the 0.25 AU radius structure found in lunar occultation $K$-band observations (Simon et al. 1987). We do not attempt to fit the inner regions and stellar photosphere with that level of detail but instead use an $800 \mathrm{~K}$ blackbody with an effective radius of $1.4 \mathrm{AU}$ to explain the remaining $80 \%$ of $M$-band emission and roughly the shape of the SED at shorter wavelengths (Fig. 9).

The disk alone is also insufficient to explain the emission beyond $\sim 55 \mu \mathrm{m}$. The single-dish line emission clearly reveals the presence of appreciable columns of material at 2.7 and $3.8 \mathrm{~km} \mathrm{~s}^{-1}(\S 4.1)$. When filling the $I S O$ beam with these columns, the emission between 55 and $180 \mu \mathrm{m}$ can be fitted at a dust temperature of $19 \mathrm{~K}$. This material provides sufficient opacity in the $10 \mu \mathrm{m}$ silicate band to turn the disk's 
emission feature into the observed absorption feature. The opacity shortward of $2 \mu \mathrm{m}$ is also sufficient to explain the observed steep drop in emission at short wavelengths. This strongly suggests that a large fraction of the 2.7 and $3.8 \mathrm{~km}$ $\mathrm{s}^{-1}$ material is located in front of Elias 29, as was found from the absorption in the ${ }^{12} \mathrm{CO}$ emission lines as well $(\S 4.1)$.

While the model of disk and foreground clouds fits the SED well, it produces a $1.3 \mathrm{~mm}$ continuum emission that is a factor of 3 larger than observed with the IRAM $30 \mathrm{~m}$ telescope at large distances from the object ( 230 mJy; Fig. 10). This may result directly from the adopted "dual-beam" mapping procedure of the IRAM $30 \mathrm{~m}$ observations, which filters out emission on the chopping scale (Motte et al. 1998; Johnstone et al. 2000a). Additionally, the model of disk and foreground predicts a source size at $1.3 \mathrm{~mm}$ that is too small (Fig. 10). The observed profile indicates that the source extends to a radius of at least $3800 \mathrm{AU}\left(25^{\prime \prime}\right)$, much larger than is realistic for a circumstellar disk. Instead, it suggests that Elias 29 and its disk are embedded in a residual cloud condensation from which the system formed. For lack of stronger constraints, we model this envelope (as we will refer to this condensation from now on) with the inside-out collapse model of Shu (1977). Earlier work (e.g., Hogerheijde \& Sandell 2000; Ceccarelli et al. 2000) has shown that this model provides an adequate description of the density and temperature of envelopes around young stellar objects. The two parameters of this model are the sound speed and the time since the collapse started, for which we take, rather arbitrarily, $0.13 \mathrm{~km} \mathrm{~s}^{-1}$ and $1.2 \times 10^{5} \mathrm{yr}$, respectively. This gives an envelope mass of $0.12 M_{\odot}$ and a radius of the "collapse expansion wave" of $6000 \mathrm{AU}$. Additional parameters are listed in Table 7. This model, combined with the disk, produces a radial emission profile that fits the measured 1.3 $\mathrm{mm}$ continuum distribution much better than the disk alone (Fig. 10). The SED below $\sim 100 \mu \mathrm{m}$ is remarkably little affected by the envelope even though with $0.12 M_{\odot}$ it is

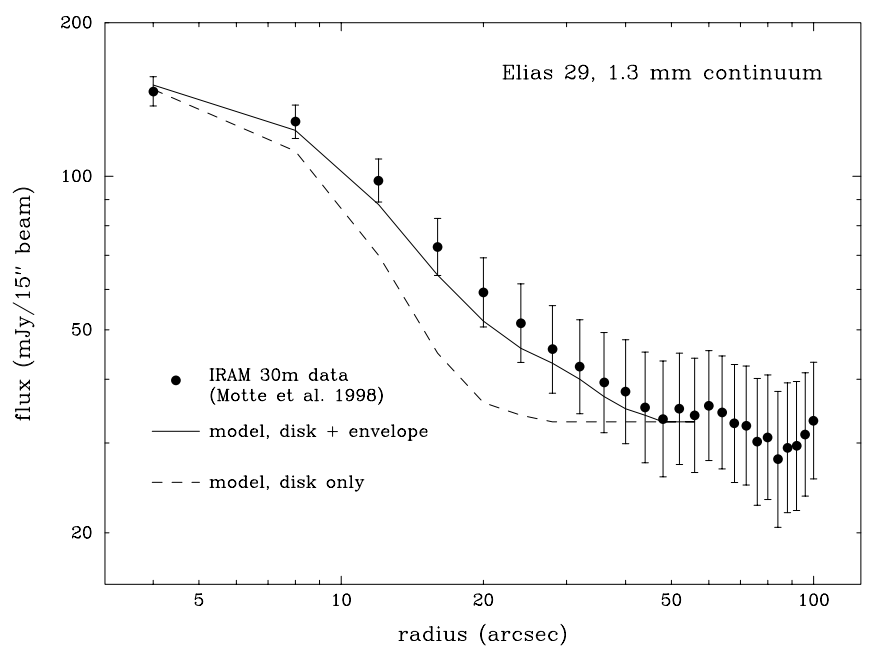

FIG. 10.-Radial intensity profile along the ridge centered on Elias 29 obtained from the $1.3 \mathrm{~mm}$ map of Motte et al. (1998) (bullets with error bars) compared to the predicted profile based on a model for Elias 29 with a disk and an envelope (solid line) and with a disk only (dashed line). Both models include a $19 \mathrm{~K}, N\left(\mathrm{H}_{2}\right)=6 \times 10^{21} \mathrm{~cm}^{-2}$ background to fit the leveling off of the emission beyond a radius of $25^{\prime \prime}-50^{\prime \prime}$. Note that the true total background column, filtered out of the IRAM $30 \mathrm{~m} 1.3 \mathrm{~mm}$ data, is a factor of 3 higher $(\S 4.2 .1)$. much more massive than the disk $\left(0.012 M_{\odot}\right.$; Table 7$)$. The material in the disk, of course, has a somewhat higher temperature range than the envelope (200-33 vs. 44-17 K, respectively), yielding significantly more infrared flux. The envelope produces only a small amount of additional absorption in the $10 \mu \mathrm{m}$ silicate band.

The parameters for the disk and envelope derived above (Table 7) maximize the contribution to the SED from the disk and minimize the mass of the envelope. There are several pieces of evidence that favor, but do not prove, this maximum-disk model above models with smaller disks. First, as for sources with directly detected disks (Hogerheijde 1998), the disk contributes about half of the $1.3 \mathrm{~mm}$ continuum flux ( $\sim 120 \mathrm{mJy}$ per $15^{\prime \prime}$ beam; foreground subtracted). Indeed, assuming that the weak $3 \mathrm{~mm}$ continuum flux within the small OVRO beam $(\S 2.2)$ originates from the disk, then the expected $1.3 \mathrm{~mm}$ disk flux would be half the single-dish flux $(F[1.3 \mathrm{~mm}]=49 \pm 14 \mathrm{mJy}$, taking spectral index 2.5; Hogerheijde 1998). Second, the above-mentioned $400 \mathrm{AU}$ radius structure, emitting thermally at $5 \mu \mathrm{m}$, may well be the surface of a large, warm disk, and third, a large face-on flared disk is the simplest way to explain the observed flat infrared SED.

Nevertheless, the evidence for a large 500 AU radius disk is not conclusive, and therefore, we also try to maximize the envelope's contribution in our model. We find that its mass can be as large as $0.33 M_{\odot}$, while the corresponding disk mass needs to be reduced to $0.002 M_{\odot}$, in part by reducing the disk's radius to $30 \mathrm{AU}$ to preferentially decrease the amount of cool material. The disk's temperature scaling does not change appreciably with respect to that used before. It must be emphasized that in this minimum disk scenario, the flatness of the infrared SED is the result of a "disk/envelope conspiracy" rather than a direct consequence of the flared disk's properties. Although the disk and envelope masses are different from those listed in Table 7, they are comparable to within the same order of magnitude, and this level of uncertainty does not affect our conclusions. In the remainder of this section we will adopt the slightly preferred values from Table 7, i.e., the maximum disk case.

\subsubsection{Emission-Line Modeling}

We use the accelerated Monte Carlo method of Hogerheijde \& van der Tak (2000) to calculate the molecular excitation and the resulting line emission in the various beams, including a realistic sampling of spatial scales recovered by the interferometer observations. Because the disk and envelope exhibit a range of densities and temperatures, we cannot use the much simpler escape probability approach here. We calculate the line intensities for three different cases (Table 8): for the disk alone, for the envelope alone, and for the combination of disk+envelope taking into account optical depth effects. The predicted line intensities are meant to be illustrative only. The general trends are robust, however. Adopting "fiducial" molecular abundances derived for the two foreground clouds (Table 5), we find that the emission of $\mathrm{C}^{18} \mathrm{O}$ observed with OVRO is overestimated by at least a factor of 5 by the disk+envelope model. This can be accounted for by adopting a depletion factor of $5-10$ for $\mathrm{CO}$ in the disk. A large $\mathrm{CO}$ depletion factor has been seen in many other disks as well (Dutrey, Guilloteau, \& Guelin 1997; Shuping et al. 2001). From the single-dish lines it can also be concluded, in particular from CS 7-6, that a signifi- 
cant depletion in the disk is needed (Table 5). Furthermore, we find that the higher excitation transitions are dominated by the disk+envelope, while the lower excitation lines can only be partially explained by this model. A fair fraction of these lines must therefore originate in the ridge.

\subsection{The Dense Ridge}

As mentioned above, Elias 29 appears to be embedded in a ridge traced by the $1.3 \mathrm{~mm}$ continuum and $\mathrm{HCO}^{+} 3-2$ line emission but slightly offset from the crest of the ridge. How much of the line emission measured in the single-dish and interferometer beams at $5.0 \mathrm{~km} \mathrm{~s}^{-1}$ can be attributed to the Elias 29 disk+envelope system and how much originates in this dense ridge? This question is relevant because it addresses the issue of how much additional column density resides in the ridge that can harbor the ice absorption bands. When we subtract the predicted emission of the disk and envelope of Elias 29 from the single-dish line fluxes at 5.0 $\mathrm{km} \mathrm{s}^{-1}$ of Table 4 , we can use the ratios of the remaining intensities to roughly constrain the conditions in the ridge. Figure $8 c$ shows the results for the $\mathrm{C}^{18} \mathrm{O} \quad 1-0 / 3-2$ and $\mathrm{HCO}^{+} 1-0 / 3-2$ lines. The density is constrained at $(4 \pm 2) \times 10^{5} \mathrm{~cm}^{-3}$, significantly higher than that of the foreground clouds, while the temperature is found to be similarly low at $15 \pm 5 \mathrm{~K}$. These parameters are consistent with the appearance of the ridge in the $1.3 \mathrm{~mm}$ and $\mathrm{HCO}^{+}$ 3-2 maps (cold and dense). The corresponding column density along the ridge is $1 \times 10^{22} \mathrm{~cm}^{-2}$, comparable to that of the largest column density foreground cloud at $3.8 \mathrm{~km} \mathrm{~s}^{-1}$. Elias 29 is offset from the maximum column density in the ridge, however, and the pencil beam traced in the infrared absorption features may contain a significantly smaller column density. The fact that the foreground clouds and, to a lesser extent, the envelope can already fit the $10 \mu \mathrm{m}$ absorption would suggest that the ridge does not contribute significantly. This conclusion does not change if we assume the higher envelope mass of $0.3 M_{\odot}$.

\subsection{Location and Thermal History of Ices toward Elias 29}

We have identified four regions of significant column density in the line of sight of Elias 29: the 2.7 and $3.8 \mathrm{~km} \mathrm{~s}^{-1}$ foreground clouds, the dense ridge, and the envelope. All of these are cold environments in which icy mantles can exist, and together they must account for the rich spectrum of ices seen with the ISO satellite (Boogert et al. 2000b). Their relative contribution to the visual extinction and ice bands depends on which fraction of the column is actually in front of Elias 29. The 2.7 and $3.8 \mathrm{~km} \mathrm{~s}^{-1}$ clouds are likely in front (§ 4.1) with columns corresponding to visual extinctions $A_{V}=2.9$ and 8.2. The visual extinction of the dense ridge, $A_{V} \leq 5.9$, is an upper limit because the ridge is somewhat displaced from Elias 29. For the envelope, $A_{V}$ is $2.5 \mathrm{mag}$ for the minimum- and 6 mag for the maximum-envelope case. These are upper limits because the system is seen at low inclination and much of the envelope material is likely not in front of Elias 29. The extinction from either ridge or envelope is, however, not negligible given the absorption seen at $5.0 \mathrm{~km} \mathrm{~s}^{-1}$ in the CO 6-5 emission line (Fig. 3). Finally, extinction by the circumstellar disk is not significant because of its low inclination.

There is observational evidence that ices in molecular clouds form only above a certain threshold extinction value

TABLE 8

Predicted Line Intensities

\begin{tabular}{|c|c|c|c|c|c|}
\hline Line & Observed $^{\mathrm{a}}$ & Envelope + Disk $^{\mathrm{b}}$ & Envelope ${ }^{\mathrm{c}}$ & Disk $^{\mathrm{d}}$ & Adopted Abundance \\
\hline \multicolumn{6}{|c|}{ Single-Dish Observations $\left(\mathrm{K} \mathrm{km} \mathrm{s}^{-1}\right)$} \\
\hline $\mathrm{C}^{18} \mathrm{O} 1-0 \ldots \ldots \ldots \ldots \ldots$ & 3.97 & 0.57 & 0.52 & 0.05 & $3.6 \times 10^{-7}$ \\
\hline $\mathrm{C}^{18} \mathrm{O} 2-1 \ldots \ldots \ldots \ldots \ldots$ & 9.50 & 3.22 & 2.64 & 0.75 & \\
\hline $\mathrm{C}^{18} \mathrm{O} 3-2 \ldots \ldots \ldots \ldots \ldots$ & 6.78 & 4.57 & 2.97 & 2.15 & \\
\hline $\mathrm{HCO}^{+} 1-0 \ldots \ldots \ldots \ldots$ & 4.26 & 1.01 & 0.99 & 0.10 & $3 \times 10^{-9}$ \\
\hline $\mathrm{HCO}^{+} 3-2 \ldots \ldots \ldots \ldots$ & 3.17 & 2.32 & 1.98 & 3.43 & \\
\hline $\mathrm{HCO}^{+} 4-3 \ldots \ldots \ldots \ldots . .$. & 0.76 & 1.51 & 0.69 & 2.87 & \\
\hline $\mathrm{H}^{13} \mathrm{CO}^{+} 1-0 \ldots \ldots \ldots$. & 0.33 & 0.04 & 0.03 & 0.01 & $4 \times 10^{-11}$ \\
\hline CS $2-1 \ldots \ldots \ldots \ldots \ldots \ldots \ldots$ & 1.72 & 0.78 & 0.76 & 0.13 & $1 \times 10^{-8}$ \\
\hline CS $5-4 \ldots \ldots \ldots \ldots \ldots \ldots \ldots$ & 0.82 & 1.49 & 0.40 & 2.14 & \\
\hline CS $7-6 \ldots \ldots \ldots \ldots \ldots \ldots \ldots$ & $<0.13$ & 4.44 & 0.11 & 4.39 & \\
\hline $\mathrm{C}^{34} \mathrm{~S} 2-1 \ldots \ldots \ldots \ldots \ldots$ & $<0.05$ & 0.07 & 0.06 & 0.02 & $4 \times 10^{-10}$ \\
\hline $\mathrm{o}-\mathrm{H}_{2} \mathrm{CO} 2_{12}-1_{11} \ldots \ldots$. & 1.03 & 1.16 & 1.13 & 0.27 & $8 \times 10^{-9}$ \\
\hline $\mathrm{o}-\mathrm{H}_{2} \mathrm{CO} 2_{11}-1_{10} \ldots \ldots$. & 0.65 & 0.86 & 0.83 & 0.31 & \\
\hline $\mathrm{o}-\mathrm{H}_{2} \mathrm{CO} 3_{12}-2_{11} \ldots \ldots$ & 0.78 & 1.0 & 0.77 & 1.66 & \\
\hline $\mathrm{p}-\mathrm{H}_{2} \mathrm{CO} 1_{01}-0_{00} \ldots \ldots$ & 0.65 & 0.19 & 0.18 & 0.04 & $2 \times 10^{-9}$ \\
\hline p- $\mathrm{H}_{2} \mathrm{CO} 2_{02}-1_{01} \ldots \ldots$. & 0.39 & 0.06 & 0.02 & 0.05 & \\
\hline $\mathrm{p}-\mathrm{H}_{2} \mathrm{CO} 3_{03}-2_{02} \ldots \ldots$ & 0.82 & 0.90 & 0.54 & 0.99 & \\
\hline $\mathrm{p}-\mathrm{H}_{2} \mathrm{CO} 3_{22}-2_{21} \ldots \ldots$ & $<0.12$ & 1.62 & 0.09 & 1.55 & \\
\hline \multicolumn{6}{|c|}{ Interferometer Observations (Jy beam ${ }^{-1}$, Image Peak per Channel) } \\
\hline $\mathrm{C}^{18} \mathrm{O} 1-0 \ldots \ldots \ldots \ldots \ldots$ & $\lesssim 0.26$ & 1.22 & 0.14 & 1.93 & \\
\hline${ }^{13} \mathrm{CO} 1-0 \ldots \ldots \ldots \ldots \ldots$ & 1.56 & 1.77 & 0.82 & 4.76 & \\
\hline $\mathrm{HCO}^{+} 1-0 \ldots \ldots \ldots \ldots . . .$. & 1.30 & 1.11 & 0.92 & 3.77 & \\
\hline
\end{tabular}

a Intensity for entire $5.0 \mathrm{~km} \mathrm{~s}^{-1}$ component, including ridge; taken from Table 4.

b Modeled intensity for disk/envelope system; no depletion assumed.

${ }^{\mathrm{c}}$ Modeled intensity envelope; infinite depletion disk.

$\mathrm{d}$ Modeled intensity disk; infinite depletion envelope. 
( $A_{\text {th }}$; Whittet et al. 1988; Tanaka et al. 1990) because of photodesorption at the cloud edges (Tielens \& Hagen 1982). Above $A_{\text {th }}$, the ice abundances grow linearly with $A_{V}$. The visual extinction can thus, in principle, be used as an indicator for $\mathrm{H}_{2} \mathrm{O}$ ice growth and destructions mechanisms. Unfortunately, the usefulness of this relation is limited by the uncertainty in $A_{\mathrm{th}}$. Measurements of a variety of sight lines in $\rho \mathrm{Oph}$ indicate $A_{\text {th }}=12$ (Teixeira \& Emerson $1999 \mathrm{~b})$, but $A_{\text {th }}<7$ toward OB stars tracing ices in foreground clouds only (Shuping et al. 2000). The $A_{V}$ values used to calibrate the aforementioned relation are highly uncertain as well (Teixeira \& Emerson 1999a). While our emission-line method has its own uncertainties (beam dilution, disk/envelope ratio), the generally used $H-K$ color method may be uncertain by a factor of 2 , for example, because of an unknown color of the intrinsic light source. Indeed, for Elias 29, a much lower $A_{V}<29$ was found from the $J-H$ color (Th. P. Greene 1998, personal communication) compared to the $H-K$ color $\left(A_{V}=47\right.$; Wilking \& Lada 1983). Nevertheless, using the relation in Teixeira \& Emerson (1999a) and assuming that the threshold extinction for $\mathrm{H}_{2} \mathrm{O}$ formation $\left(A_{\mathrm{th}}=12\right)$ applies only once for all clouds combined, we find that the total $A_{V}<23$ corresponds to $N\left(\mathrm{H}_{2} \mathrm{O}\right)<11 \times 10^{17} \mathrm{~cm}^{-2}$, which is $<30 \%$ of the observed ice column density (Boogert et al. 2000b). In this picture, the observed ice column is thus much higher than that expected from the total visual extinction along the line of sight. Given the above-mentioned uncertainties in the ice column versus $A_{V}$ and $A_{\text {th }}$ relation, the most reliable conclusion that can be drawn from this is that $\mathrm{H}_{2} \mathrm{O}$ ice sublimation does not play an important role in the Elias 29 line of sight.

From a different perspective, the low temperature of the clouds along the line of sight (Table 5) indicates that indeed no sublimation or other forms of thermal processing (e.g., crystallization) of polar, $\mathrm{H}_{2} \mathrm{O}$-rich ices can take place. A similar conclusion was previously drawn from the (low) farinfrared color index, which is found to be a good indicator of thermal processing of ices (Boogert et al. 2000a; van der Tak et al. 2000). Even the temperature of the warmest absorbing component, the inner part of the envelope ( $\sim 45$ $\mathrm{K})$, is less than the $\mathrm{H}_{2} \mathrm{O}$ crystallization $(\sim 60 \mathrm{~K})$ and sublimation temperatures $(\sim 90 \mathrm{~K})$. This explains the observed lack of crystallization signatures in the absorption band profiles of solid $\mathrm{H}_{2} \mathrm{O}$ and $\mathrm{CO}_{2}$ (Boogert et al. 2000b).

Thermal processing must have played a role for apolar, CO-rich ices toward Elias 29, however. The solid $\mathrm{CO} / \mathrm{H}_{2} \mathrm{O}$ ratio is $5 \%$, which is a factor of 5 less than in several other lines of sight within the $\rho$ Oph cloud (Shuping et al. 2000). We find that Elias 29 has heated its envelope sufficiently $(17-44 \mathrm{~K} ; \S 4.2)$ to evaporate CO-rich ices $\left(T_{\text {subl }} \sim 15-20 \mathrm{~K}\right.$; Tielens et al. 1991) but preserve $\mathrm{H}_{2} \mathrm{O}$-rich ices. The envelope, however, harbors only a small fraction of the ices, and it is unlikely that this $38 L_{\odot}$ object can heat ices located beyond the envelope, such as the dense ridge and the two foreground clouds. Nevertheless, the temperatures of the gas and dust in the quiescent foreground clouds are similar to, or slightly higher (Table 5) than, the sublimation temperature of apolar ices. Several known luminous B stars and the Sco OB2 association play an important role in heating the dust in the $\rho$ Oph cloud (Greene \& Young 1989). The differences of the solid $\mathrm{CO} / \mathrm{H}_{2} \mathrm{O}$ ratio within $\rho$ Oph may thus be explained by the location of the ices with respect to these luminous heating sources. Bright external heating sources are absent in the Taurus molecular cloud, and indeed, the $\mathrm{CO} / \mathrm{H}_{2} \mathrm{O}$ ratios are significantly larger in that cloud (Teixeira \& Emerson 1999a).

\subsection{Depletion}

The depletion factor of $\mathrm{CO}$ [defined as solid/ (gas + solid)] amounts to a few percent, assuming the ices are present in several of the clouds toward Elias 29 [using $N($ CO-ice $)=1.7 \times 10^{17} \mathrm{~cm}^{-2}$; Boogert et al. 2000b]. Direct measurements of solid $\mathrm{CO}$ yield larger depletion factors in the quiescent Taurus molecular cloud $(8 \%-40 \%$; Chiar et al. 1995). Indirect measurements, which rely on gas-phase abundances only, found $\mathrm{CO}$ depletion factors as high as $90 \%$ toward deeply embedded Class 0 sources such as NGC 1333: IRAS 4A and 4B (Blake et al. 1995). We will compare gas-phase abundances of other molecules toward Elias 29 with those of Class 0 sources as well as the well-studied dense cloud TMC 1 (Table 5).

Even when taking the uncertainty of gas-phase abundances into account, we find that $\mathrm{CO}, \mathrm{CS}, \mathrm{H}_{2} \mathrm{CO}$, and possibly $\mathrm{HCO}^{+}$are an order of magnitude more depleted in the Class 0 sources compared to the clouds in front of Elias 29. This can be ascribed to the much larger densities and low dust temperatures in the outer regions $(\gtrsim 700 \mathrm{AU})$ of these Class 0 objects and thus shorter depletion timescales (Blake et al. 1995). Depletion is much lower in the TMC 1 cloud compared to Class 0 objects (Table 5). In fact, the $\mathrm{H}_{2} \mathrm{CO}$ and $\mathrm{CH}_{3} \mathrm{OH}$ abundances are an order of magnitude larger than toward Elias 29. Whether these species are depleted toward Elias 29 cannot be answered directly because the upper limit to the ice abundances are typically a few times $10^{-6}$ [Boogert et al. 2000b; taking $\left.N\left(\mathrm{H}_{2}\right)=2.9 \times 10^{22} \mathrm{~cm}^{-2}\right]$. This is 4 and 2 orders of magnitude larger than the gas-phase $\mathrm{CH}_{3} \mathrm{OH}$ and $\mathrm{H}_{2} \mathrm{CO}$ abundances, respectively, and demonstrates that a direct determination of depletion factors using infrared ice bands suffers from a lack of sensitivity. Alternatively, the difference in gas-phase $\mathrm{CH}_{3} \mathrm{OH}$ and $\mathrm{H}_{2} \mathrm{CO}$ abundances between these clouds may have its origin in different chemical evolutionary states, such as was found within the TMC 1 cloud itself for several carbon-bearing species (Pratap et al. 1997). Indeed, $\mathrm{H}_{2} \mathrm{CO}$ abundances of several other quiescent and star-forming cores $\left[(2-10) \times 10^{-9}\right.$; Dickens \& Irvine $1999]$ are in much better agreement with Elias 29.

In some Class 0 sources, the $\mathrm{CH}_{3} \mathrm{OH}$ and $\mathrm{H}_{2} \mathrm{CO}$ abundances and temperatures are larger than in our comparison source NGC 1333, IRAS 4, possibly because they are more evolved (van Dishoeck et al. 1995; Blake et al. 1995). This warm gas originates from evaporated ices that are enriched in hydrogenated molecules by grain surface reactions during the collapse of the cold envelope. Shocks (van Dishoeck et al. 1995) and thermal evaporation (Ceccarelli et al. 2001) in the inner envelope have been proposed as ice desorption mechanisms, which can enhance the $\mathrm{H}_{2} \mathrm{CO}$ abundance at radii smaller than $150 \mathrm{AU}$ by 2 orders of magnitude. The single-dish observations of Elias 29 do not show evidence of such warm gas, as is illustrated in Figure 11 by comparing the $\mathrm{CH}_{3} \mathrm{OH}$ spectrum with the Class 0 source IRAS 16293-2422. This may well be an effect of the larger column and accretion rate of the younger Class 0 objects. Infrared absorption line studies do reveal, however, that significant amounts of warm $\mathrm{CO}$ and $\mathrm{H}_{2} \mathrm{O}$ are present on small scales near the protostar, partly related to the outflow, and probably partly present in the accreting disk's atmosphere (Boogert et al. 2000b; A. C. A. Boogert et al. 2002, in prepa- 


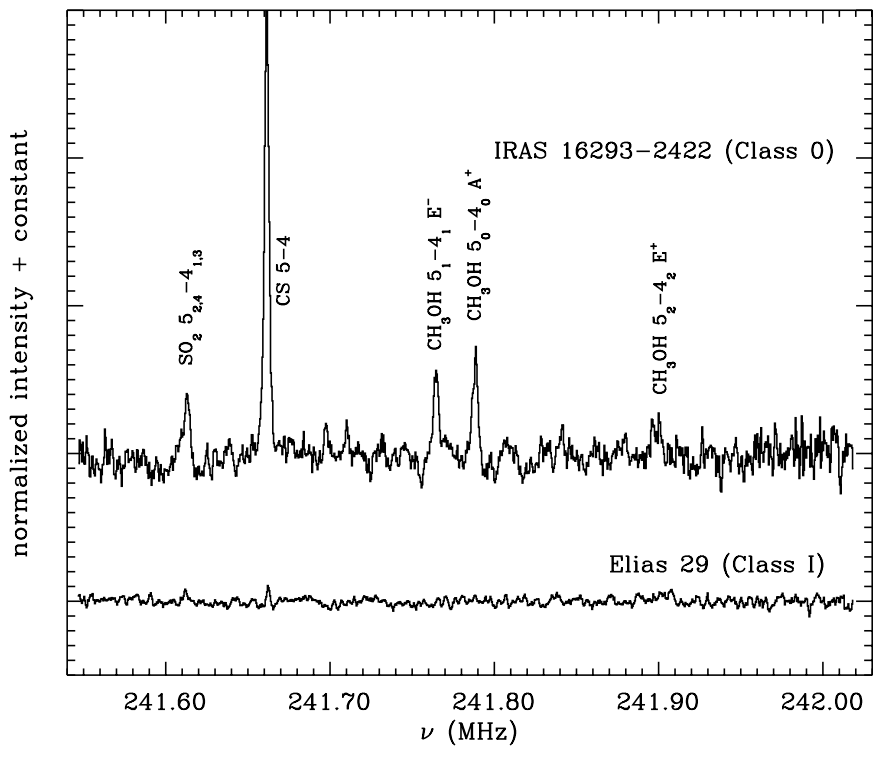

FIG. 11.-Low-resolution spectrum showing the enormous difference in line intensity between the Class 0 object IRAS 16293-2422 and the Class I object Elias 29 in all the lines of $\mathrm{SO}_{2}, \mathrm{CS}$, and $\mathrm{CH}_{3} \mathrm{OH}$.

ration). Emission from this gas is severely diluted in the large single-dish millimeter wave beams and thus difficult to detect.

Finally, the $\mathrm{HCO}^{+}$abundance toward Elias 29 is comparable to that found toward Class 0 objects but an order of magnitude lower compared to TMC 1. The importance of depletion is more difficult to establish because the $\mathrm{HCO}^{+}$ abundance is highly dependent on local cosmic-ray flux and electron density. Even in the direction of Elias 29 the $\mathrm{HCO}^{+}$ abundance varies dramatically. Although the $\mathrm{HCO}^{+} 1-0$ and CS 2-1 lines have similar critical densities, the ridge (at $5.0 \mathrm{~km} \mathrm{~s}^{-1}$ ) is much more prominent in $\mathrm{HCO}^{+}$than in $\mathrm{CS}$ with respect to the 2.7 and $3.8 \mathrm{~km} \mathrm{~s}^{-1}$ foreground clouds (Fig. 3). We stress, however, that the prominent appearance of the ridge in the $\mathrm{HCO}^{+} 3-2$ map (Fig. 1) is an effect of both increased $\mathrm{HCO}^{+}$abundance and increased density: the ridge is weaker in the column density tracer $\mathrm{C}^{18} \mathrm{O}$ and stronger in the density tracer CS relative to the foreground clouds.

\section{SUMMARY, CONCLUSIONS, AND FUTURE WORK}

We have analyzed infrared and millimeter wave line and continuum observations to construct a model of the Class I protostar Elias 29 and its environment. This model has to contain a number of different components (summarized in Fig. 7): a disk to account for the $\sim 2-50 \mu \mathrm{m}$ SED, an envelope contributing to the emission at $1.3 \mathrm{~mm}$ (in particular, its size), a dense ridge from which Elias 29 may have condensed, and foreground material that provides most of the extinction. Elias 29 can then be well described by a $500 \mathrm{AU}$ radius face-on flared disk with a mass of $0.012 M_{\odot}$, embedded in a 6000 AU radius, $0.12 M_{\odot}$ envelope. This large disk provides the simplest explanation for the observed flat SED, weakly detected $3 \mathrm{~mm}$ continuum emission, and $400 \mathrm{AU}$ radius $5 \mu \mathrm{m}$ thermal continuum emission. The present data does not, however, fully exclude models with smaller disks. The minimum possible disk has a $30 \mathrm{AU}$ radius and a mass of $0.002 M_{\odot}$ surrounded by an envelope of $0.33 M_{\odot}$. In this case the combination of disk and envelope emission produces a flat SED.

The entire system is embedded in a long, dense, cold, and $\mathrm{HCO}^{+}$-rich ridge. Elias 29 is slightly offset from the crest of this ridge. In front of the disk, envelope, and ridge system are two foreground clouds at a few kilometers per second lower radial velocities that cover the entire field of view. The large column of the foreground clouds, corresponding to $A_{V} \sim 11$, may be responsible for the "Class I" appearance of Elias 29, which would otherwise appear as a T Tauri or Herbig Ae/Be star (i.e., optically visible). These same foreground clouds are also the most likely repository of most of the ices seen along the line of sight $(\sim 70 \%)$. The low temperature of the foreground clouds explains the observed absence of crystallized ices and the presence of large abundances of polar, $\mathrm{H}_{2} \mathrm{O}$-rich ices; i.e., thermal processing did not play a major role for the ices toward Elias 29. The foreground cloud temperature $(25 \pm 15 \mathrm{~K})$ could, however, be high enough to explain the low abundance of apolar, volatile CO-rich ices, presumably due to the proximity of a number of luminous B-type stars. The important question as to whether the ices in the disk or envelope have experienced thermal processing, as is seen in the envelopes of massive objects (Boogert et al. 2000a; van der Tak et al. 2000), cannot be addressed given the large column of foreground material and the face-on nature of the system.

This work shows the value of spectrally and spatially resolved information offered by single-dish and interferometric molecular gas observations in interpreting infrared ISO satellite observations of ices along a pencil beam. It shows that, at least for the $\rho \mathrm{Oph}$ cloud, it is crucial to disentangle the different physical components along the line of sight since otherwise incorrect conclusions may be derived, for example, on the origin and evolution of interstellar and circumstellar ices.

Follow-up observations with sensitive submillimeter interferometers (e.g., ALMA) or high-frequency $(>400$ $\mathrm{GHz}$ ) single-dish telescopes with small beams will clearly provide essential information on the structure, abundances, and depletion factor of species in the disk and outflow of Elias 29, and its relationship to the envelope and ridge. In turn, such studies would provide invaluable information on the initial conditions of planet formation.

We thank Remo Tilanus, Goeran Sandell, and Fred Baas for carrying out part of the JCMT observations in service mode. The research of F. M. and A. C. A. B. at the Caltech Submillimeter Observatory is funded by the NSF through contract AST 99-80846. The research of M. R. H. is supported by the Miller Institute for Basic Research in Science.
Blake, G. A., Sandell, G., van Dishoeck, E. F., Groesbeck, T. D., Mundy, L. G., \& Aspin, C. 1995, ApJ, 441, 689

Bohlin, R. C., Savage, B. D., \& Drake, J. F. 1978, ApJ, 224, 132

Boogert, A. C. A., et al. 2000a, A\&A, 353, 349

Boogert, A. C. A., Tielens, A. G. G. M., Ceccarelli, C., Boonman, A. M. S., van Dishoeck, E. F., Keane, J. V., Whittet, D. C. B., \& de Grauuw, Th. 2000 b, A\&A, 360, 683
REFERENCES

Ceccarelli, C., Castets, A., Caux, E., Hollenbach, D., Loinard, L., Molinari, S., \& Tielens, A. G. G. M. 2000, A\&A, 355, 1129

Ceccarelli, C., Loinard, L., Castets, A., Tielens, A. G. G. M., Caux, E., Lefloch, B., \& Vastel, C. 2001, A\&A, 372, 998

Chandler, C. J., \& Richer, J. S. 2000, ApJ, 530, 851

Chen, H., Myers, P. C., Ladd, E. F., \& Wood, D. O. S. 1995, ApJ, 445, 377

Chiang, E. I., \& Goldreich, P. 1997, ApJ, 490, 368 
Chiang, E. I., \& Goldreich, P. 1999, ApJ, 519, 279

Chiar, J. E., Adamson, A. J., Kerr, T. H., \& Whittet, D. C. B. 1995, ApJ, 455,234

Dickens, J. E., \& Irvine, W. M. 1999, ApJ, 518, 733

Dullemond, C. P., Dominik, C., \& Natta, A. 2001, ApJ, 560, 957

Dutrey, A., Guilloteau, S., \& Guelin, M. 1997, A\&A, 317, L55

Elias, J. H. 1978, ApJ, 224, 857

Greene, Th. P., \& Young, E. T. 1989, ApJ, 339, 258

Hogerheijde, M. R. 1998, Ph.D. thesis, Rijksuniv. Leiden

Hogerheijde, M. R., \& Sandell, G. 2000, ApJ, 534, 880

Hogerheijde, M. R., \& van der Tak, F. F. S. 2000, A\&A, 362, 697

Imanishi, K., Koyama, K., \& Tsuboi, Y. 2001, ApJ, 557, 747

Jansen, D. J. 1995, Ph.D. thesis, Rijksuniv. Leiden

Johnstone, D., Wilson, C. D., Moriarty-Schieven, G., GiannakopoulouCreighton, J., \& Gregersen, E. 2000a, ApJS, 131, 505

Johnstone, D., Wilson, C. D., Moriarty-Schieven, G., Joncas, G., Smith, G., Gregersen, E., \& Fich, M. 2000b, ApJ, 545, 327

Kutner, M. L., \& Ulich, B. L. 1981, ApJ, 250, 341

Lacy, J. H., Knacke, R., Geballe, T. R., \& Tokunaga, A. T. 1994, ApJ, 428, L69

Loren, R. B. 1989, ApJ, 338, 902

Mangum, J. G. 1993, PASP, 105, 117

1999, User's Manual for the NRAO $12 \mathrm{~m}$ Millimeter-Wave

Telescope, NRAO Internal Rep.

Motte, F., André, Ph., \& Neri, R. 1998, A\&A, 336, 150

Ohishi, M., \& Kaifu, N. 1998, Faraday Discuss., 109, 205

Ossenkopf, V., \& Henning, Th. 1994, A\&A, 291, 943

Pratap, P., Dickens, J. E., Snell, R. L., Miralles, M. P., Bergin, E. A., Irvine, W. M., \& Schloerb, F. P. 1997, ApJ, 486, 862

Ristorcelli, I., et al. 1999, in Les Houches, Session XI, Solid Interstellar Matter: The ISO Revolution, ed. L. d'Hendecourt, C. Joblin, \& A. Jones (Les Ulis: EDP Sciences), 49
Shu, F. 1977, ApJ, 214, 488

Shuping, R. Y., Chiar, J. E., Snow, Th., \& Kerr, Th. 2001, ApJ, 547, L161

Shuping, R. Y., Snow, Th., Chiar, J. E., \& Kerr, Th. 2000, ApJ, 529, 932

Simon, M., Howell, R. R., Longmore, A. J., Wilking, B. A., Peterson, D. M., \& Chen, W. P. 1987, ApJ, 320, 344

Tanaka, M., Sato, S., Nagata, T., \& Yamamoto, T. 1990, ApJ, 352, 724

Teixeira, T. C., \& Emerson, J. P. 1999a, A\&A, 351, 292 1999b, A\&A, 351, 303

Tielens, A. G. G. M., \& Charnley, S. B. 1997, Origins Life Evol. Biosphere, 27,23

Tielens, A. G. G. M., \& Hagen, W. 1982, A\&A, 114, 245

Tielens, A. G. G. M., Tokunaga, A. T., Geballe, T. R., \& Baas, F. 1991, ApJ, 381, 181

van der Tak, F. F. S., van Dishoeck, E. F., Evans, N. J. II, \& Blake, G. A. 2000, ApJ, 537, 283

van Dishoeck, E. F., \& Blake, G. A. 1998, ARA\&A, 36, 317

van Dishoeck, E. F., Blake, G. A., Jansen, D. J., \& Groesbeck, T. D. 1995, ApJ, 447, 760

Whittet, D. C. B. 1974, MNRAS, 168, 371

Whittet, D. C. B., Bode, M. F., Longmore, A. J., Adamson, A. J., McFadzean, A. D., Aitken, D. K., \& Roche, P. F. 1988, MNRAS, 233, 321

Whittet, D. C. B., et al. 1998, ApJ, 498, L159

Wilking, B. A., \& Lada, C. J. 1983, ApJ, 274, 698

Wilson, T. L., \& Rood, R. T. 1994, ARA\&A, 32, 191

Zinnecker, H., Perrier, C., \& Chelli, A. 1988, in ESO Conf. Proc. 29, NOAO-ESO Conference on High-Resolution Imaging by Interferometry, ed. F. Merkle (Garching: ESO), 505

Zinnecker, H., Webster, A. S., \& Geballe, T. R. 1985, in Nearby Molecular Clouds, ed. G. Serra (Berlin: Springer), 81 